\title{
Investigation of inhibition effect of daidzein on osteosarcoma cells based on experimental validation and systematic pharmacology analysis
}

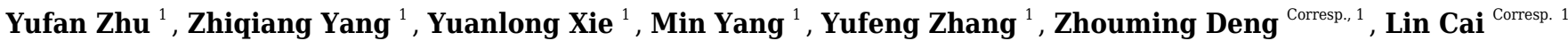 \\ ${ }^{1}$ Department of Spine Surgery \& Musculoskeletal Tumor, Zhongnan Hospital of Wuhan University, Wuhan, Hubei, People's Republic of China \\ Corresponding Authors: Zhouming Deng, Lin Cai \\ Email address: dengzhouming@whu.edu.cn, orthopedics@whu.edu.cn
}

Objective: This study aims to explore the effect of daidzein, which is a natural isoflavone compound mainly extracted from soybeans, on osteosarcoma and the potential molecular mechanism.

Material and methods: $143 \mathrm{~B}$ and U2OS osteosarcoma cells were treated with gradient concentrations of daidzein, and MTT assay was used to determine the cell proliferation capacity and IC ${ }_{50}$. Hoechst 33342 staining and Annexin V-FITC/PI detection were used to determine apoptosis. Cell cycle was analyzed by flow cytometry, and migration ability were detected by transwell assays and scratch wound assay. An osteosarcoma xenograft mice model was applied to investigate the effect of daidzein on osteosarcoma in vivo. Systematic pharmacology and molecular modeling analysis were applied to predict the target of daidzein to osteosarcoma, and the target Src was verified by western blotting. We also observed the effect of daidzein on cell proliferation and apoptosis of Src-overexpressing osteosarcoma cells.

Results: In vitro, daidzein significantly inhibited 143B and U2OS osteosarcoma cell proliferation and migration, and induced cell cycle arrest. In vivo, Daidzein exerts antitumor effects in osteosarcoma xenograft mice. After systematic screening and analysis, Src-MAPK signaling pathway was predicted as the highest-ranked pathway. Western blot demonstrated that daidzein inhibited phosphorylation of the Src-ERK pathway in osteosarcoma cells. And overexpression of Src could partially reverse the inhibitory effects of daidzein on osteosarcoma cell proliferation.

Conclusion: Daidzein exerts an antitumor effect on osteosarcoma, the mechanism may be through the Src-ERK pathway. 


\section{Investigation of inhibition effect of daidzein on}

2 osteosarcoma cells based on experimental validation

3 and systematic pharmacology analysis

Yufan Zhu ${ }^{1}$, Zhiqiang Yang ${ }^{1}$, Yuanlong Xie ${ }^{1}$, Min Yang ${ }^{1}$, Yufeng Zhang ${ }^{1}$, Zhouming Deng ${ }^{1}$, Lin $\mathrm{Cai}^{1}$

7

8

${ }^{1}$ Department of Spine Surgery \& Musculoskeletal Tumor, Zhongnan Hospital of Wuhan University, Wuhan, Hubei, PR China

Corresponding Author:

Lin $\mathrm{Cai}^{1}$

\#169, East Lake Road, Wuchang District, Wuhan, Hubei, 430072, PR China

Email address: orthopedics@whu.edu.cn

Zhouming Deng ${ }^{1}$

Email address: dengzhouming@whu.edu.cn

\section{Abstract}

Objective: This study aims to explore the effect of daidzein, which is a natural isoflavone compound mainly extracted from soybeans, on osteosarcoma and the potential molecular mechanism.

Material and methods: 143B and U2OS osteosarcoma cells were treated with gradient concentrations of daidzein, and MTT assay was used to determine the cell proliferation capacity and $\mathrm{IC}_{50}$. Hoechst 33342 staining and Annexin V-FITC/PI detection were used to determine apoptosis. Cell cycle was analyzed by flow cytometry, and migration ability were detected by transwell assays and scratch wound assay. An osteosarcoma xenograft mice model was applied to investigate the effect of daidzein on osteosarcoma in vivo. Systematic pharmacology and molecular modeling analysis were applied to predict the target of daidzein to osteosarcoma, and the target Src was verified by western blotting. We also observed the effect of daidzein on cell proliferation and apoptosis of Src-overexpressing osteosarcoma cells.

Results: In vitro, daidzein significantly inhibited 143B and U2OS osteosarcoma cell proliferation and migration, and induced cell cycle arrest. In vivo, Daidzein exerts antitumor effects in osteosarcoma xenograft mice. After systematic screening and analysis, Src-MAPK signaling pathway was predicted as the highest-ranked pathway. Western blot demonstrated that daidzein inhibited phosphorylation of the Src-ERK pathway in osteosarcoma cells. And cell proliferation. 
39 Conclusion: Daidzein exerts an antitumor effect on osteosarcoma, the mechanism may be

40 through the Src-ERK pathway.

41 Introduction

42 Osteosarcoma is the most common primary tumor of bone in children and adolescent. The 5-year

43 survival rate of patients with localized osteosarcoma is approximately $70 \%$, whereas the overall

44 survival rate of patients with metastatic or recurrent disease is less than $20 \%$ (Harrison et al.

45 2018). Based on historical data, over $80 \%$ of patients will develop metastasis following resection

46 of the primary tumor alone, and even with the addition of chemotherapy to primary tumor

47 resection, approximately one-third of patients presenting with localized disease will subsequently

48 develop pulmonary metastases (Khanna et al. 2014). Standard treatment modalities consist of

49 combining surgical excision with chemotherapy, which has changed very little over the past 30

50 years (Gianferante et al. 2017; Li et al. 2020; Luu \& Viloria-Petit 2020). The three-drug

51 chemotherapy regimen of cisplatin, doxorubicin, and methotrexate (MAP) forms the backbone

52 therapy, and the response to induction chemotherapy correlates with patient outcome

53 (Kleinerman 2016). Due to the clinical heterogeneity of osteosarcoma, the selection of treatment

54 options and effective drug combinations remain limited. It is generally accepted that plants are

55 regarded as valuable resources for the development of drugs to treat many diseases, including

56 cancer (Thomford et al. 2018). Some natural active ingredients display strong antitumor activity;

57 in addition, compared with chemotherapy, they have fewer adverse effects and no resistance to

58 chemotherapy. Therefore, there is an urgent requirement to examine novel and more effective

59 natural compounds for the treatment of osteosarcoma.

60 Flavonoids are phenolic compounds with more than 6000 family members that widely exist in a

61 large number of plants and fungi. Isoflavone is a natural flavonoid that plays the role of

62 phytoestrogens in mammals and has become a research hotspot in recent years (Zhang et al.

63

64

2018). Daidzein, whose chemical composition is 7,4-dihydroxyisoflavone, belongs to the

65

isoflavone family. It is the most frequently ingested and deeply studied phytoestrogen (Jin et al.

66

2010). In previous studies, daidzein has been proven to have antitumor effects on a variety of tumors, including breast cancer (Bao et al. 2014; Magee et al. 2014), bladder cancer (He et al. 2016), prostate cancer (Singh-Gupta et al. 2010; Yu et al. 2003), colon cancer (Liang et al. 2018), endometrial carcinogenesis (Lian et al. 2001), melanoma (Wang et al. 2002), neuroblastoma (Lo et al. 2007), and choriocarcinoma (Zheng et al. 2017). Recently, Luisa

71

72

73

74

75

76

77

78
Salvatori et al. reported that daidzein inhibited proliferation and cell cycle progression and promoted apoptosis in a U2OS human osteosarcoma cell line in vitro (Salvatori et al. 2009), but there has been no research on the related mechanism. In addition, no other study has demonstrated that daidzein is capable of inhibiting osteosarcoma. Therefore, we carried out an in-depth study on the effect and mechanisms of daidzein against osteosarcoma. Systemic pharmacology, proposed by Andrew L. Hopkins, is an emerging discipline that integrates systems biology and pharmacology (Hopkins 2008). It provides a new way to explore the mechanism of drugs from the molecular and cellular levels to the tissue and organism levels (Berger \& Iyengar 2009). After verifying the inhibitory effects of daidzein on osteosarcoma in 
vitro and in vivo, we applied systemic pharmacology theory and molecular docking analysis to identify the intersection of potential targets between osteosarcoma and daidzein, and selected the key target, Src, and its downstream MAPK pathway. Finally, a rescue experiment was carried out to validate the mechanisms of action. The methodological flowchart of this study is shown in Figure 1 .

\section{Materials \& Methods}

\section{Reagents}

Daidzein [7-hydroxy-3- (4-hydroxyphenyl) chromen-4-one] was purchased from Aladdin Industrial, Inc. (Shanghai, China), which with a purity over 98\%. It was dissolved in DMSO at a final concentration of $100 \mathrm{mM}$ in medium and stored at $-20^{\circ} \mathrm{C}$. Dimethyl sulfoxide (DMSO) was obtained from Sinopharm Chemical Reagent Co., Ltd. (China).

\section{Cell culture}

Human osteosarcoma cell lines (143B and U2OS) and human osteoblast cell line hFOB 1.19 were obtained from the China Centre for Type Culture Collection (Wuhan, China). 143B cells were cultured in Roswell Park Memorial Institute (RPMI) 1640 medium supplemented with 10\% heat - inactivated FBS (Gibco) and $1 \%$ antibiotics in a $37^{\circ} \mathrm{C}$ incubator with $5 \% \mathrm{CO}_{2}$. U2OS cells were cultured in McCoy's 5A medium (modified) when hFOB 1.19 was cultured in $\alpha$-MEM (HyClone) and the other conditions were the same as those used for 143B cells.

\section{Cell proliferation analysis}

Cells were plated in 96-well plates at a density of 2000 cells per well and incubated at $37^{\circ} \mathrm{C}$ for $12 \mathrm{~h}$. Then, the cells were treated for the indicated time periods with different concentrations of daidzein diluted in medium containing $10 \%$ FBS. After incubation, the supernatants were removed, and MTT solution (Beyotime, Shanghai, China) was added to each well (100 $\mu \mathrm{L}$ per well). Following incubation at $37^{\circ} \mathrm{C}$ in a dark environment for an additional $4 \mathrm{~h}$, the supernatants were removed again, and $200 \mu \mathrm{L}$ DMSO was added to each well to solubilize formazan. After 10 min, the 96-well plates were placed in a microplate reader, and the absorbance was recorded at $570 \mathrm{~nm}$. The data came from three independent experiments, each with six copies. The mean cell viability of the treated cells was used to calculate the survival fractions.

\section{Colony formation assay}

The cells were evenly seeded in a 6-well plate at a density of 500 cells per well. After $24 \mathrm{~h}$, the cells were treated with different concentrations of daidzein for approximately 7 days until the cells grew into visible colonies. Then, the supernatant was discarded, and the cells were washed with PBS three times. Colonies were then fixed with $4 \%$ paraformaldehyde for $30 \mathrm{~min}$ and stained with $1 \%$ crystal violet for $10 \mathrm{~min}$. The number of colonies (more than 50 cells/colony) was counted and quantified.

\section{Cell apoptosis and cycle detection}

Following exposure to daidzein for a specified period, osteosarcoma cells were collected for apoptosis and cycle detection. Hoechst 33342 (Beyotime, Shanghai, China) was used to detect cell apoptosis by observing the cell morphology. After osteosarcoma cells were treated, the medium was discarded, and the cells were washed twice with PBS and then fixed with sufficient 
$1194 \%$ paraformaldehyde at $4^{\circ} \mathrm{C}$ for $10 \mathrm{~min}$. Then, sufficient Hoechst 33342 staining solution was 120 added to cover the cells. After incubation in the dark for $10 \mathrm{~min}$, the cells were washed with PBS

121 and then immediately observed and imaged by fluorescence microscopy. In addition, cell

122 apoptosis and cell cycle progression were analyzed by flow cytometry. Cell apoptosis of human

123 osteosarcoma cells was analyzed based on Annexin V-FITC/propidium iodide (PI) dual staining

124 when the cell cycle was assessed by staining cells with $20 \mu \mathrm{g} / \mathrm{mL}$ PI.

125 6. Migration ability detection

126 The migration ability of osteosarcoma cells was detected by Transwell migration assay and 127 scratch wound assay. The Transwell experimental procedure was as follows: Approximately

$1285 \times 10^{4}$ cells suspended in $100 \mu \mathrm{L}$ serum-free RPMI 1640 or McCoy's $5 \mathrm{~A}$ medium were added to 129 each upper chamber, and $600 \mu \mathrm{L}$ of medium containing $10 \%$ FBS was added to the lower 130 chamber. Cells were cultured at $37^{\circ} \mathrm{C}$ for $24 \mathrm{~h}$, fixed with $4 \%$ paraformaldehyde for 30 min and 131 stained with $1 \%$ crystal violet for $10 \mathrm{~min}$. Cotton swabs were used to remove the unmigrated 132 cells on the upper surface of the membrane, and the cells that migrated on the lower surface of 133 the film were counted. As for scratch wound assays, cells were plated in a 6-well plate at a 134 density of $5 \times 10^{5}$ cells per well and then incubated at $37^{\circ} \mathrm{C}$ until almost complete confluence. 135 Three parallel wounds were made with a $10 \mu \mathrm{L}$ pipette tip per well. Cells were cultured in 136 serum-free medium supplemented with different concentrations of daidzein. At $0 \mathrm{~h}$ and $24 \mathrm{~h}$, 137 photos were taken at the same position under an optical microscope, and the wound width of 138 each group was compared.

\section{7. Establishment of a nude mouse osteosarcoma xenograft model}

140 Twelve four-week-old male BALB/c nude mice obtained from Hubei Experimental Animal

141 Research Center (Hubei, China) were used for animal experiments. 143B cells were digested and 142 suspended in cold PBS at a final concentration of $1 \times 10^{7}$ cells per $\mathrm{mL}$. In this model, a total of $143100 \mu \mathrm{L}$ of the prepared cell suspension was injected in the right subaxillary area subcutaneously.

1447 days after tumor inoculation, when the volume of the tumor was approximately $100 \mathrm{~mm}^{3}$, 145 daidzein $(20 \mathrm{mg} / \mathrm{kg})$ was administered by tail vein injection every 2 days in the experimental 146 group ( $\mathrm{n}=6$ ), while the control group $(\mathrm{n}=6)$ was injected with an equal volume of DMSO. The 147 injection treatment lasted for 9 days, and the tumor volume and mouse body weight were 148 measured every 3 days. Tumor volume $\left(\mathrm{mm}^{3}\right)$ was calculated using the following equation: 149 length $\times$ width $^{2} \times 0.5$. On day 16 , mice were sacrificed by dislocation of cervical vertebra after 150 anesthesia with sodium pentobarbital (100mg/kg body weight, tail vein injection), and the 151 xenografts were dissected and fixed with $4 \%$ polyformaldehyde for $\mathrm{H} \& \mathrm{E}$ staining analysis.

153

154 When the tumor diameter exceeded $20 \mathrm{~mm}$ or rupture and infection occurred, as well as when treatment was ineffective after systemic infection occurred, mice were euthanized using the method described above.

This in vivo study was conducted according to the guidelines of the Declaration of Helsinki, and approved by the Institutional Review Board (or Ethics Committee) of Institutional Animal Care 157 and Use Committee, Wuhan University Center for Animal Experiment (protocol code WP2020158 08106. On August 10th, 2020). 


\section{8. Target identification and network construction}

160

161

162

163

164

165

166

167

168

169

170

171

172

173

174

175

176

177

178

179

180

181

182

183

184

185

186

187

188

189

190

191

192

193

194

195

196

197

198

The osteosarcoma-related targets were collected and combined from the following databases: DigSeE (http://210.107.182.61/geneSearch/), DisGeNET (http://www.disgenet.org/), GeneCards (https://www.genecards.org/), KEGG disease (https://www.kegg.jp/kegg/disease/), MalaCards (http://www.malacards.org/), and Phenopedia (https://phgkb.cdc.gov/PHGKB/startPagePhenoPedia.action). The validated targets of daidzein were extracted from PubChem (https://pubchem.ncbi.nlm.nih.gov/) and PharmMapper (http://www.lilabecust.cn/pharmmapper/). Common targets of osteosarcoma and daidzein were discovered and retained by VENNY 2.1.0 (https://bioinfogp.cnb.csic.es/tools/venny/) for further analysis. A protein-protein interaction (PPI) network was constructed by STRING (https://string$\mathrm{db}$. org/) and visualized by Cytoscape software. The Database for Annotation, Visualization and Integrated Discovery (DAVID; http://david.abcc.ncifcrf.gov/) was used to perform GO and pathway enrichment analyses, and Cytoscape software (Version 3.8.1) was used to construct the target-function network.

\section{Molecular docking studies}

To identify the potential interaction between Src and daidzein, molecular docking simulations were conducted with AutoDock Vina (version 1.1.2) to predict their preferred binding conformation. PubChem was referenced for the SDF file of daidzein, and the SDF file was transformed into a PDB file by Open Babel 2.3.2. The crystal structure of human Src was derived from the Protein Data Bank (PDB) database (http://www.rcsb.org, PDB code: 2H8H). Before molecular docking analysis, PYMOL 2.3.4 software was used to remove the water molecules as well as ligands from the receptor protein, and AutodockTools software was used to balance the charge and affix hydrogen atoms to the receptor protein. Grid Option was used to adjust the number of grid points in each direction, the center of the binding pocket and the spacing between the grid points. The centroid of the daidzein molecule in the crystal structures of the complex was defined as the binding site. AutoDock Vina software was used for molecular docking of the Src protein and daidzein molecule, and the results were output in the form of affinity. The affinity is obtained by calculating the space effect, repulsion, hydrogen bond, hydrophobic interaction and molecular flexibility of the complex, which is an important indicator to measure whether the ligand can effectively bind to the receptor molecule. The lower the value, the better the binding effect.

\section{Analysis of proteins in osteosarcoma cells treated with daidzein}

Cold PBS was used to wash the adherent osteosarcoma cells cultured in 6-well plates three times. RIPA buffer containing a proteinase inhibitor (Beyotime, Shanghai, China) was used to extract total protein from osteosarcoma cells. Osteosarcoma cells were scraped with a cell scraper, dissolved in buffer, and transferred to a $1.5 \mathrm{~mL}$ EP tube with a pipette. The above samples were treated with an ultrasonic cell breaker, with ultrasonic treatment for $3 \mathrm{~s}$ and an interval time of $10 \mathrm{~s}$, which was repeated three times. Then, the lysates were centrifuged at $4^{\circ} \mathrm{C}$ and $13000 \mathrm{~g}$ for $15 \mathrm{~min}$. In this way, the protein composition was separated and diluted for denaturation. Protein samples were subjected to SDS-PAGE and then transferred to PVDF membranes. The blocking 
199

200

201

202

203

204

205

206

207

208

209

210

211

212

213

214

215

216

217

218

219

220

221

222

223

224

225

226

227

228

229

230

231

232

233

234

235

236

237

238

solution was prepared by dissolving $5 \%$ skim milk powder in TBST, and the membrane was put into the solution and blocked for $1 \mathrm{~h}$. The membranes were then incubated with the following rabbit anti-human polyclonal primary antibodies overnight: anti-Src (ab109381), anti-p-Src (ab40660, phospho Y418), anti-JNK (ab179461), anti-p-JNK (ab76572, phospho Y185+Y185+Y223), anti-ERK (ab184699), anti-p-ERK (ab201015, phospho T202+T185), antip38 (ab178867, phospho T180), anti-p-p38 (ab195049, phospho T180+Y182) and anti-GAPDH (ab9485) (Abcam, Shanghai, China). GAPDH expression was used as a loading control. After washing three times, the membranes were incubated with the corresponding goat anti-rabbit secondary antibodies (Abcam, Shanghai, China). A Western blot visualization (ECL kit, Pierce, USA) and analysis system (Bio-Rad) was used to detect chemiluminescence.

\section{Construction of Src-overexpressing osteosarcoma cell lines by lentivirus transfection} For Src overexpression, 143B and U2OS cells were transfected with $4 \mu \mathrm{g}$ of pMSCV-Flag or pMSCV-Flag-Src using Lipofectamine 3000 Transfection Reagent (Thermo Scientific, USA) according to the manufacturer's instructions. Empty virus vectors were used as negative controls. The primers were 5'-tgttcggaggcttcaactcc-3' (F) and 5'-gacatccaccttcctcgtgt-3' (R). After 72h of transfection, the expression of the green fluorescent protein EGFP in cells was observed under a fluorescence microscope, and stably transfected cells were established by the puromycin (1.5 $\mathrm{ng} / \mu \mathrm{L}$ ) screening method. The rescue experiment was performed based on stably transfected cells.

\section{Statistical analysis}

The results are expressed as the mean \pm standard deviation (SD) from at least three independent experiments. Student's t-test was used to compared the difference between two groups while, one-way analysis of variance (ANOVA) was used to analyze statistical differences between more than two groups. GraphPad Prism software (version 7.04) was used for statistical analysis. A statistically significant difference was defined as $\mathrm{p}<0.05$.

\section{Results}

\section{Daidzein inhibits the proliferation of 143B and U2OS osteosarcoma cells}

Firstly, we explored the effect of daidzein on proliferation of 143B and U2OS osteosarcoma cells. 143B and U2OS cells were treated with gradient concentrations of daidzein $(0,10,20,50$, 100,200 or $500 \mu \mathrm{mol} / \mathrm{L}$ ). After 48 or $72 \mathrm{~h}$ treatment, MTT assay was performed to detect the cell proliferation capacity. As shown in Fig.2A-B, daidzein significantly inhibited the proliferation capacity of osteosarcoma cells in a dose- and time-dependent manner. According to the absorbance curve, the $48 \mathrm{~h} 50 \%$ inhibiting concentration $\left(\mathrm{IC}_{50}\right)$ of daidzein on 143B and $\mathrm{U} 2 \mathrm{OS}$ cells are $63.59 \mu \mathrm{mol} / \mathrm{L}$ and $125 \mu \mathrm{mol} / \mathrm{L}$. Meanwhile, we treated normal human hFOB 1.19 osteoblasts with the same daidzein dose in 48 and $72 \mathrm{~h}$ (Fig. 1C). According to the analysis results of $\mathrm{IC}_{50}$ (Fig. 1D), daidzein had a much weaker inhibitory effect on the proliferation of $\mathrm{hFOB}$ cells compared with osteosarcoma cells (Student's t-test, $\mathrm{p}<0.05$ ). Thus, daidzein should be considered relatively safe when applied clinically. Then, we set three concentration treatment groups (143B: $20 \mu \mathrm{mol} / \mathrm{L}$ for the low concentration group, $48 \mathrm{~h} \mathrm{IC}$ s0 $_{50}$ group, and $200 \mu \mathrm{mol} / \mathrm{L}$ for 
239 the high concentration group; U2OS: $50 \mu \mathrm{mol} / \mathrm{L}$ for the low concentration group, $48 \mathrm{~h} \mathrm{IC} \mathrm{C}_{50}$ group, 240 and $200 \mu \mathrm{mol} / \mathrm{L}$ for the high concentration group) for the following experiments. And colony 241 formation assay results also showed that daidzein significantly inhibited the colony formation

242 ability of 143B and U2OS osteosarcoma cells (Fig.2E-F). Thus, daidzein may inhibits the 243 proliferation of 143B and U2OS osteosarcoma cells in a dose- and time-dependent manner.

244 2. Daidzein induces apoptosis and cell cycle arrest in 143B and U2OS osteosarcoma cells

245

246

247

248

249

250

251

252

253

254

255

256

257

258

259

260

261

262

263

264

265

266

267

268

269

270

271

272

273

274

275

276

277

278 Hoechst 33342 staining and Annexin V-FITC/PI dual staining detection kits were used to determine the effect of daidzein on apoptosis 143B and U2OS cells. The results indicated that $24 \mathrm{~h}$ of treatment with daidzein (at low, $\mathrm{IC}_{50}$, and high concentrations) considerably increased apoptosis in a dose-dependent manner compared to that of the control group. The results of the flow cytometric analysis showed that the early and late apoptosis rate of daidzein treatment group (at low, $\mathrm{IC}_{50}$, and high concentrations) were significantly lower than that of the control group (Fig.3A-B). Next, we determined whether daidzein was involved in the cell cycle of osteosarcoma cells. According to the cell cycle distribution analyzed by flow cytometry, we found that daidzein increased the percentage of $\mathrm{S}$ phase cells but decreased the percentage of $\mathrm{G}_{0} / \mathrm{G}_{1}$ phase cells in 143B and U2OS cells (Fig.3C-D). These results suggest that daidzein may induce apoptosis and cell cycle arrest in 143B and U2OS osteosarcoma cells.

3. Daidzein suppresses migration ability of and 143B and U2OS osteosarcoma cells We also explored the influence of daidzein on migration ability of osteosarcoma. 143B and U2OS cells were pretreated with daidzein for $24 \mathrm{~h}$ before the Transwell assays. Daidzein significantly suppressed cell migration in 143B and U2OS cells, as shown in Fig.3E. Moreover, scratch wound assay revealed that the cell mobility rates in daidzein treatment group were significantly decreased when compared with the control group (Fig.3G). Therefore, daidzein can suppress migration ability of and 143B and U2OS osteosarcoma cells.

\section{Daidzein effectively suppresses osteosarcoma growth in xenograft mice model}

To investigate the effect of daidzein on osteosarcoma in vivo, an osteosarcoma xenograft mice model was established by subcutaneous implantation of 143B cells in BALB/c nude mice. As shown in Fig.4, the volume and weight of the tumors and tumor growth rate in the daidzeintreated group significantly decreased when compared with those in the control group. H\&E staining of the tumor revealed that the number of necrotic cells in the daidzein-treated group was significantly higher than that in the control group (Figure 4E). Furthermore, the body weight of the nude mice was not significantly affected (Figure 4B), indicating that there was little systemic toxicity of daidzein in vivo.

5. Target prediction of daidzein on osteosarcoma through systemic pharmacology analysis To reveal the mechanism of daidzein regulating biological process of osteosarcoma cells, systemic pharmacology analysis was performed on PharmMapper platform. A total of 243 human genes associated with daidzein were predicted by the PharmMapper platform (Table S1). As shown in Figure 5A, a "daidzein-daidzein targets" network was constructed. Osteosarcomarelated genes were collected and combined from DigSeE, DisGeNET, GeneCards, KEGG disease, MalaCards, and Phenopedia (Table S2). After intersecting the two gene sets (Figure 5B),

Peerj reviewing PDF | (2021:03:59437:1:0:NEW 2 Jul 2021) 
279 there were 133 common targets of osteosarcoma and daidzein through which daidzein might play 280 a role in regulating the progression of osteosarcoma. After that, 133 target genes associated with 281 daidzein and osteosarcoma were imported into the STRING database for PPI network 282 construction. As shown in Figure 5C and Table S3, there were 133 nodes and 295 interaction 283 edges (interaction score $>0.9$ ). To analyze the PPI diagram, the analysis tool in Cytoscape was 284 applied, and the top 30 hub genes were identified and are shown in Figure 5D. Among the top 30 285 hub genes, Src was the node that interacted with the most numerous edges, suggesting that Src

286

287

288

289

290

291

292

293

294

295

296

297

298

299

300

301

302

303

304

305

306

307

308

309

310

311

312

313

314

315

316

317

318 may play a vital role of daidzein in regulating biological process of osteosarcoma.

\section{Src protein may be the target of daidzein}

In order to explore whether daidzein can effectively combine with Src and play a regulatory role, we assessed the potential interaction between daidzein and Src through molecular modeling and docking simulation. The 3D structure of daidzein was obtained from the PubChem database (Figure 5E). After adjustment and analysis, the grid size was set to $40 \times 40 \times 40$, and we obtained an affinity of $-9.1 \mathrm{kcal} / \mathrm{mol}$. As shown in Figure $5 \mathrm{~F}$, hydrophobic interactions were formed between daidzein and the following amino acid residues: Ile336, Thr338, Met314, Val323, Phe405, Ala403, Leu393, Asp404, Val281 and Ala293. In brief, these results suggest a strong potential of direct binding between daidzein and Src. Thereafter, we employed GO annotation for the functions of the key target genes. The top 20 highly enriched functional terms are shown in Figure 6A. The key target genes could regulate multiple biological processes, including steroid hormone receptor activity, nuclear receptor activity, ligand-activated transcription factor activity, etc. The KEGG results indicated that these key target genes were involved in multiple signaling pathways, and the top 20 pathways are shown in Figure 6B. Among them, the MAPK signaling pathway was the highest-ranked pathway, with the highest gene ratio as well as the third minimum adjusted P-value (less than $1 \mathrm{e}^{-07}$ ). According to previous studies, the MAPK (JNK/ERK/p38) signaling pathway is a downstream molecule of Src kinase (Cui et al. 2017; Gao et al. 2019). Therefore, we further predicted that the Src and MAPK pathways may be involved in the regulation of daidzein on biological process of osteosarcoma.

\section{Daidzein inhibits phosphorylation of the Src and ERK signaling pathways osteosarcoma cells}

According to the results of previous systemic pharmacology analysis and molecular docking studies, we detected the protein expression and phosphorylation levels of Src, JNK, ERK and p38 to elucidate the molecular mechanisms by which daidzein affects osteosarcoma cells. As shown in Figure 6C-D, daidzein treatment decreased the expression of p-Src and p-ERK, but the expression levels of Src, JNK, p-JNK, ERK, p38 and p-p38 were not significantly changed. Therefore, we determined that Src and ERK phosphorylation was suppressed in daidzein-treated cells, whereas JNK and p38 phosphorylation was not altered.

\section{Overexpression of Src rescues inhibitory effect of daidzein on osteosarcoma cells}


319 As shown in Fig.7B-C, overexpression of Src partially restituted the phosphorylation of Src and 320 ERK in osteosarcoma cells treated with daidzein. Moreover, according to MTT assay (Figure 321 7D) and apoptosis analysis by flow cytometry (Figure 7E-F), decreased viability and in 322 osteosarcoma cells treated with daidzein was partially reversed by overexpression of Src.

323 Therefore, it suggests that daidzein may exert anti-osteosarcoma efficiency through the inhibition 324 of the Src kinase-mediated ERK pathway.

325

326

327

328

329

330

331

332

333

334

335

336

337

338

339

340

341

342

343

344

345

346

347

348

349

350

351

352

353

354

355

356

357

\section{Discussion}

Daidzein is often found in beans such as soybeans, sweet red beans, and kidney beans. Previous studies have shown that it has antioxidative, anti-inflammatory, and anti-aging activities (Ahmad et al. 2016; Choi et al. 2012; Liu et al. 2009; Tomar et al. 2020; Yi et al. 2019; Yu et al. 2020; Živanović et al. 2019). Additionally, daidzein has garnered interest as a natural compound with antitumor effects against a variety of tumors. Several previous studies have investigated the antitumor effects and mechanisms of daidzein. For example, in colon cancer, daidzein induces apoptosis of tumor cells by inhibiting the accumulation of lipid droplets (Liang et al. 2018). In bladder cancer, daidzein exerts antitumor activity via inhibition of the FGFR3 pathway (He et al. 2016). Additionally, daidzein has also been shown to enhance the anticancer effect of topotecan and reverse BCRP-mediated drug resistance in breast cancer (Guo et al. 2019), and it inhibits lung metastasis in mice induced by B16F-10 melanoma cells (Menon et al. 1998). In malignant glioma, treatment with subtoxic doses of daidzein in combination with TRAIL induces rapid apoptosis in glioma cells because daidzein overcomes TRAIL resistance by modulating the expression of the intrinsic apoptotic inhibitor bcl-2 (Siegelin et al. 2009).

As a kind of nonreceptor protein tyrosine kinase, Src is an important oncogenic biological factor in a variety of malignancies, and the active form of Src is phosphorylated Src (p-Src) (Dehm \& Bonham 2004). Src interacts with several protein-tyrosine kinase receptors at the plasma membrane, including EGFR, erbB2, c-Met, PDGFR, IGFR and FGFR. These protein-tyrosine kinase receptors are dysregulated or mutated in a variety of human malignancies, including and not limited to NSCLC, breast cancer, pancreatic cancer, colorectal cancer, bladder cancer, prostate cancer, kidney cancer, ovarian cancer and sarcoma and so on (Roskoski 2015). Src and that of its protein-tyrosine kinase receptors interact with each other. In addition to protein tyrosine kinases, Src-family kinases are also regulated by integrin receptors, G-protein coupled receptors, antigen- and Fc-coupled receptors, cytokine receptors, and steroid hormone receptors (Thomas \& Brugge 1997). As the central hub of multiple signaling pathways, Src is involved in the amplification cascade of the intracellular network regulation system, whose downstream pathways include FAK, Akt, Stat 3 and MAPK. Src is also involved in the regulation of bone formation and remodeling and may mediate skeletal metastasis of breast, prostate, and lung cancers (Roskoski 2015). Our previous clinical studies have shown that Src/p-Src are potential biomarkers of osteosarcoma, which could promote the progression or metastasis of osteosarcoma and may lead to a poorer prognosis in clinical patients (Hu et al. 2015). 
358

359

360

361

362

363

364

365

366

367

368

369

370

371

372

373

374

375

376

377

378

379

380

381

382

383

384

385

386

387

388

389

390

391

392

393

394

395

396

397

398

399

In modern civilization, the spectrum of human diseases has changed greatly, from infectious diseases to complex diseases such as tumors, cardiovascular diseases, and diabetes. The occurrence and development of complex diseases often involve multiple genes and signaling pathways in the regulatory network of the organism. Systemic pharmacology aims at biological networks and analyzes the relationship among the drugs, targets and diseases in these networks (Yuan et al. 2017), which is consistent with the treatment concept of complex diseases: to carry out new drug research and drug mechanistic research. The large amount of experimental material and reagents used in previous screening studies has been saved due to the availability of data from the Internet for analysis, thereby greatly saving time and expense.

\section{Conclusions}

In conclusion, through a combination of systemic pharmacology, molecular docking and experimental validation, this study provides powerful proof that daidzein has little negative effect on normal cells and exerts an antitumor effect through the Src-ERK axis, which suggests that daidzein could act as a potential osteosarcoma chemotherapy agent. However, this study has its limitations. First, owing to limitations of time and funds, instead of experimentally validating all target molecules and pathways screened by the network pharmacological analysis, we chose the one with the highest match possibility. Second, we only examined the anti-osteosarcoma effects of daidzein, but we did not validate the mechanism in vivo. In addition, depending on the different cell types and stimuli, MAPKs have a dual role since they can act as activators or inhibitors of apoptosis (Yue \& López 2020). Therefore, further indepth study is needed on the specific mechanism by which daidzein inhibits ERK phosphorylation and thus causes apoptosis in osteosarcoma cells. In addition, more basic and clinical studies are needed to establish the role of daidzein in the treatment of osteosarcoma.

\section{Acknowledgements}

The authors thank Dr. Xiaobin Zhu and Bin Ren from the Department of Spine Surgery \& Musculoskeletal Tumor, Zhongnan Hospital of Wuhan University, China for assisting in experimental design.

\section{References}

Ahmad S, Alam K, Hossain MM, Fatima M, Firdaus F, Zafeer MF, Arif Z, Ahmed M, and Nafees KA. 2016. Anti-arthritogenic and cardioprotective action of hesperidin and daidzein in collageninduced rheumatoid arthritis. Mol Cell Biochem 423:115-127. 10.1007/s11010-016-2830-y

Bao C, Namgung H, Lee J, Park HC, Ko J, Moon H, Ko HW, and Lee HJ. 2014. Daidzein suppresses tumor necrosis factor- $\alpha$ induced migration and invasion by inhibiting hedgehog/Gli1 signaling in human breast cancer cells. J Agric Food Chem 62:3759-3767. 10.1021/jf500231t

Berger SI, and Iyengar R. 2009. Network analyses in systems pharmacology. Bioinformatics 25:24662472. 10.1093/bioinformatics/btp465

Choi EY, Jin JY, Lee JY, Choi JI, Choi IS, and Kim SJ. 2012. Anti-inflammatory effects and the underlying mechanisms of action of daidzein in murine macrophages stimulated with Prevotella intermedia lipopolysaccharide. J Periodontal Res 47:204-211. 10.1111/j.1600-0765.2011.01422.x 
400

401

402

403

404

405

406

407

408

409

410

411

412

413

414

415

416

417

418

419

420

421

422

423

424

425

426

427

428

429

430

431

432

433

434

435

436

437

438

439

440

441

442

443

444

445

446

447

448

449

Cui S, Wang J, Wu Q, Qian J, Yang C, and Bo P. 2017. Genistein inhibits the growth and regulates the migration and invasion abilities of melanoma cells via the FAK/paxillin and MAPK pathways. Oncotarget 8:21674-21691. 10.18632/oncotarget.15535

Dehm SM, and Bonham K. 2004. SRC gene expression in human cancer: the role of transcriptional activation. Biochem Cell Biol 82:263-274. 10.1139/o03-077

Gao Z, Zhao GS, Lv Y, Peng D, Tang X, Song H, and Guo QN. 2019. Anoikis-resistant human osteosarcoma cells display significant angiogenesis by activating the Src kinase-mediated MAPK pathway. Oncol Rep 41:235-245. 10.3892/or.2018.6827

Gianferante DM, Mirabello L, and Savage SA. 2017. Germline and somatic genetics of osteosarcoma connecting aetiology, biology and therapy. Nat Rev Endocrinol 13:480-491. 10.1038/nrendo.2017.16

Guo J, Wang Q, Zhang Y, Sun W, Zhang S, Li Y, Wang J, and Bao Y. 2019. Functional daidzein enhances the anticancer effect of topotecan and reverses BCRP-mediated drug resistance in breast cancer. Pharmacol Res 147:104387. 10.1016/j.phrs.2019.104387

Harrison DJ, Geller DS, Gill JD, Lewis VO, and Gorlick R. 2018. Current and future therapeutic approaches for osteosarcoma. Expert Rev Anticancer Ther 18:39-50. 10.1080/14737140.2018.1413939

He Y, Wu X, Cao Y, Hou Y, Chen H, Wu L, Lu L, Zhu W, and Gu Y. 2016. Daidzein exerts antitumor activity against bladder cancer cells via inhibition of FGFR3 pathway. Neoplasma 63:523531. 10.4149/neo_2016_405

Hopkins AL. 2008. Network pharmacology: the next paradigm in drug discovery. Nat Chem Biol 4:682690. 10.1038/nchembio. 118

Hu C, Deng Z, Zhang Y, Yan L, Cai L, Lei J, and Xie Y. 2015. The prognostic significance of Src and p-Src expression in patients with osteosarcoma. Med Sci Monit 21:638-645. $10.12659 / \mathrm{msm} .892803$

Jin S, Zhang QY, Kang XM, Wang JX, and Zhao WH. 2010. Daidzein induces MCF-7 breast cancer cell apoptosis via the mitochondrial pathway. Ann Oncol 21:263-268. 10.1093/annonc/mdp499

Khanna C, Fan TM, Gorlick R, Helman LJ, Kleinerman ES, Adamson PC, Houghton PJ, Tap WD, Welch DR, Steeg PS, Merlino G, Sorensen PH, Meltzer P, Kirsch DG, Janeway KA, Weigel B, Randall L, Withrow SJ, Paoloni M, Kaplan R, Teicher BA, Seibel NL, Smith M, Uren A, Patel SR, Trent J, Savage SA, Mirabello L, Reinke D, Barkaukas DA, Krailo M, and Bernstein M. 2014. Toward a drug development path that targets metastatic progression in osteosarcoma. Clin Cancer Res 20:4200-4209. 10.1158/1078-0432.ccr-13-2574

Kleinerman E. 2016. Maximum benefit of chemotherapy for osteosarcoma achieved-what are the next steps? Lancet Oncol 17:1340-1342. 10.1016/s1470-2045(16)30270-4

Li Z, Li X, Xu D, Chen X, Li S, Zhang L, Chan MTV, and Wu WKK. 2020. An update on the roles of circular RNAs in osteosarcoma. Cell Prolif:e12936. 10.1111/cpr.12936

Lian Z, Niwa K, Tagami K, Hashimoto M, Gao J, Yokoyama Y, Mori H, and Tamaya T. 2001. Preventive effects of isoflavones, genistein and daidzein, on estradiol-17beta-related endometrial carcinogenesis in mice. Jpn J Cancer Res 92:726-734. 10.1111/j.1349-7006.2001.tb01154.x

Liang YS, Qi WT, Guo W, Wang CL, Hu ZB, and Li AK. 2018. Genistein and daidzein induce apoptosis of colon cancer cells by inhibiting the accumulation of lipid droplets. Food \& nutrition research 62. 10.29219/fnr.v62.1384

Liu MH, Lin YS, Sheu SY, and Sun JS. 2009. Anti-inflammatory effects of daidzein on primary astroglial cell culture. Nutr Neurosci 12:123-134. 10.1179/147683009x423274

Lo FH, Mak NK, and Leung KN. 2007. Studies on the anti-tumor activities of the soy isoflavone daidzein on murine neuroblastoma cells. Biomed Pharmacother 61:591-595. 10.1016/j.biopha.2007.08.021

Luu AK, and Viloria-Petit AM. 2020. Targeting Mechanotransduction in Osteosarcoma: A Comparative Oncology Perspective. Int J Mol Sci 21. 10.3390/ijms21207595

Peer) reviewing PDF | (2021:03:59437:1:0:NEW 2 Jul 2021) 
450

451

452

453

454

455

456

457

458

459

460

461

462

463

464

465

466

467

468

469

470

471

472

473

474

475

476

477

478

479

480

481

482

483

484

485

486

487

488

489

490

491

492

493

494

495

496

497

498

Magee PJ, Allsopp P, Samaletdin A, and Rowland IR. 2014. Daidzein, R-(+)equol and S-(-)equol inhibit the invasion of MDA-MB-231 breast cancer cells potentially via the down-regulation of matrix metalloproteinase-2. Eur J Nutr 53:345-350. 10.1007/s00394-013-0520-z

Menon LG, Kuttan R, Nair MG, Chang YC, and Kuttan G. 1998. Effect of isoflavones genistein and daidzein in the inhibition of lung metastasis in mice induced by B16F-10 melanoma cells. Nutr Cancer 30:74-77. 10.1080/01635589809514644

Roskoski R, Jr. 2015. Src protein-tyrosine kinase structure, mechanism, and small molecule inhibitors. Pharmacol Res 94:9-25. 10.1016/j.phrs.2015.01.003

Salvatori L, Caporuscio F, Coroniti G, Starace G, Frati L, Russo MA, and Petrangeli E. 2009. Down-regulation of epidermal growth factor receptor induced by estrogens and phytoestrogens promotes the differentiation of U2OS human osteosarcoma cells. J Cell Physiol 220:35-44. $10.1002 / j \mathrm{cp} .21724$

Siegelin MD, Gaiser T, Habel A, and Siegelin Y. 2009. Daidzein overcomes TRAIL-resistance in malignant glioma cells by modulating the expression of the intrinsic apoptotic inhibitor, bcl- 2 . Neurosci Lett 454:223-228. 10.1016/j.neulet.2009.03.031

Singh-Gupta V, Zhang H, Yunker CK, Ahmad Z, Zwier D, Sarkar FH, and Hillman GG. 2010. Daidzein effect on hormone refractory prostate cancer in vitro and in vivo compared to genistein and soy extract: potentiation of radiotherapy. Pharm Res 27:1115-1127. 10.1007/s11095-0100107-9

Thomas SM, and Brugge JS. 1997. Cellular functions regulated by Src family kinases. Annu Rev Cell Dev Biol 13:513-609. 10.1146/annurev.cellbio.13.1.513

Thomford NE, Senthebane DA, Rowe A, Munro D, Seele P, Maroyi A, and Dzobo K. 2018. Natural Products for Drug Discovery in the 21st Century: Innovations for Novel Drug Discovery. Int J Mol Sci 19. 10.3390/ijms19061578

Tomar A, Kaushik S, Khan SI, Bisht K, Nag TC, Arya DS, and Bhatia J. 2020. The dietary isoflavone daidzein mitigates oxidative stress, apoptosis, and inflammation in CDDP-induced kidney injury in rats: Impact of the MAPK signaling pathway. J Biochem Mol Toxicol 34:e22431. $10.1002 / \mathrm{jbt} .22431$

Wang HZ, Zhang Y, Xie LP, Yu XY, and Zhang RQ. 2002. Effects of genistein and daidzein on the cell growth, cell cycle, and differentiation of human and murine melanoma cells(1). J Nutr Biochem 13:421-426. 10.1016/s0955-2863(02)00184-5

Yi Y, Adrjan B, Li J, Hu B, and Roszak S. 2019. NMR studies of daidzein and puerarin: active antioxidants in traditional Chinese medicine. J Mol Model 25:202. 10.1007/s00894-019-4090-8

Yu L, Blackburn GL, and Zhou JR. 2003. Genistein and daidzein downregulate prostate androgenregulated transcript-1 (PART-1) gene expression induced by dihydrotestosterone in human prostate LNCaP cancer cells. J Nutr 133:389-392. 10.1093/jn/133.2.389

Yu Z, Yang L, Deng S, and Liang M. 2020. Daidzein ameliorates LPS-induced hepatocyte injury by inhibiting inflammation and oxidative stress. Eur J Pharmacol 885:173399. 10.1016/j.ejphar.2020.173399

Yuan H, Ma Q, Cui H, Liu G, Zhao X, Li W, and Piao G. 2017. How Can Synergism of Traditional Medicines Benefit from Network Pharmacology? Molecules 22. 10.3390/molecules22071135

Yue J, and López JM. 2020. Understanding MAPK Signaling Pathways in Apoptosis. Int J Mol Sci 21. 10.3390/ijms21072346

Zhang Q, Xie H, Chen D, Yu B, Huang Z, Zheng P, Mao X, Yu J, Luo Y, Luo J, and He J. 2018. Dietary Daidzein Supplementation During Pregnancy Facilitates Fetal Growth in Rats. Mol Nutr Food Res 62:e1800921. 10.1002/mnfr.201800921

Zheng W, Sun R, Yang L, Zeng X, Xue Y, and An R. 2017. Daidzein inhibits choriocarcinoma proliferation by arresting cell cycle at G1 phase through suppressing ERK pathway in vitro and in vivo. Oncol Rep 38:2518-2524. 10.3892/or.2017.5928

PeerJ reviewing PDF | (2021:03:59437:1:0:NEW 2 Jul 2021) 
Živanović J, Jarić I, Ajdžanović V, Mojić M, Miler M, Šošić-Jurjević B, Milošević V, and Filipović 500

B. 2019. Daidzein upregulates anti-aging protein Klotho and $\mathrm{NaPi} 2 \mathrm{a}$ cotransporter in a rat model 501 of the andropause. Ann Anat 221:27-37. 10.1016/j.anat.2018.08.001 


\section{Figure 1}

The methodological flowchart of this study.

This study firstly experimentally demonstrated the therapeutic effect of daidzein on osteosarcoma, then through the exploration of network pharmacology and molecular docking technology, key molecules and pathways were targeted and experimentally validated. Website links: DigSeE (http://210.107.182.61/geneSearch/) DisGeNET (http://www.disgenet.org/), GeneCards (https://www.genecards.org/) KEGG disease (https://www.kegg.jp/kegg/disease/) MalaCards (http://www.malacards.org/) Phenopedia (https://phgkb.cdc.gov/PHGKB/startPagePhenoPedia.action) PubChem (https://pubchem.ncbi.nlm.nih.gov/) PharmMapper (http://www.lilabecust.cn/pharmmapper/) VENNY 2.1.0 (https://bioinfogp.cnb.csic.es/tools/venny/). 

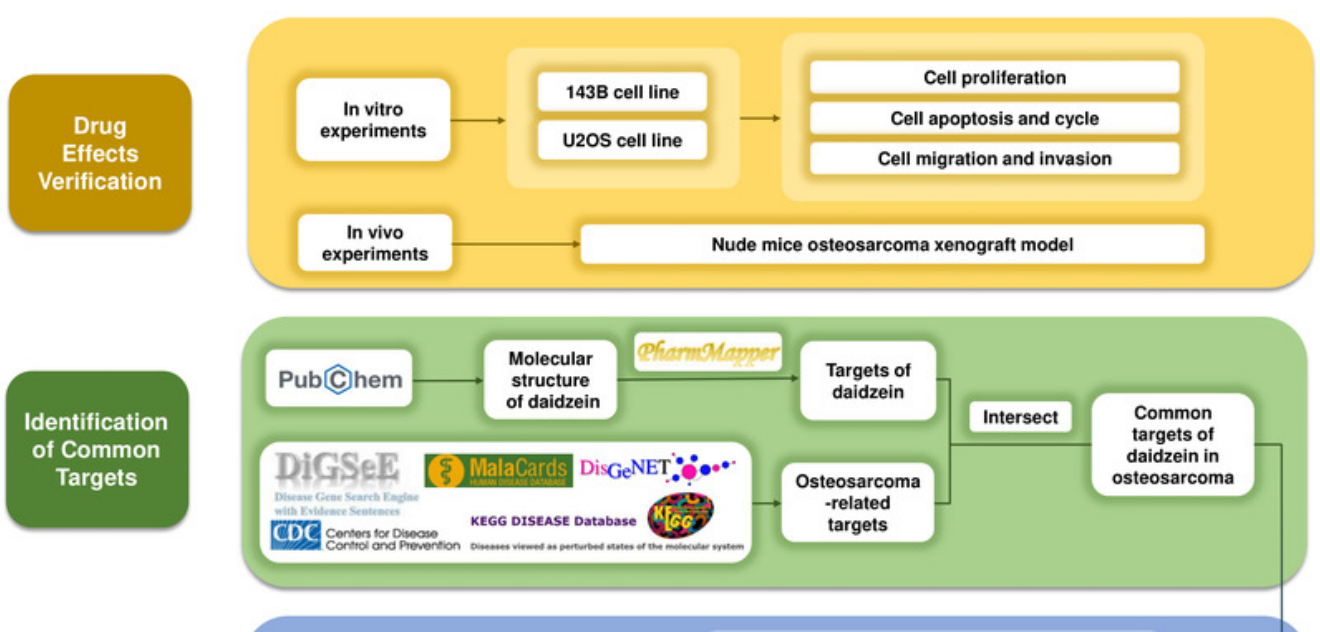

Network

Construction

and Analysis

Experimental

Mechanism

Verification

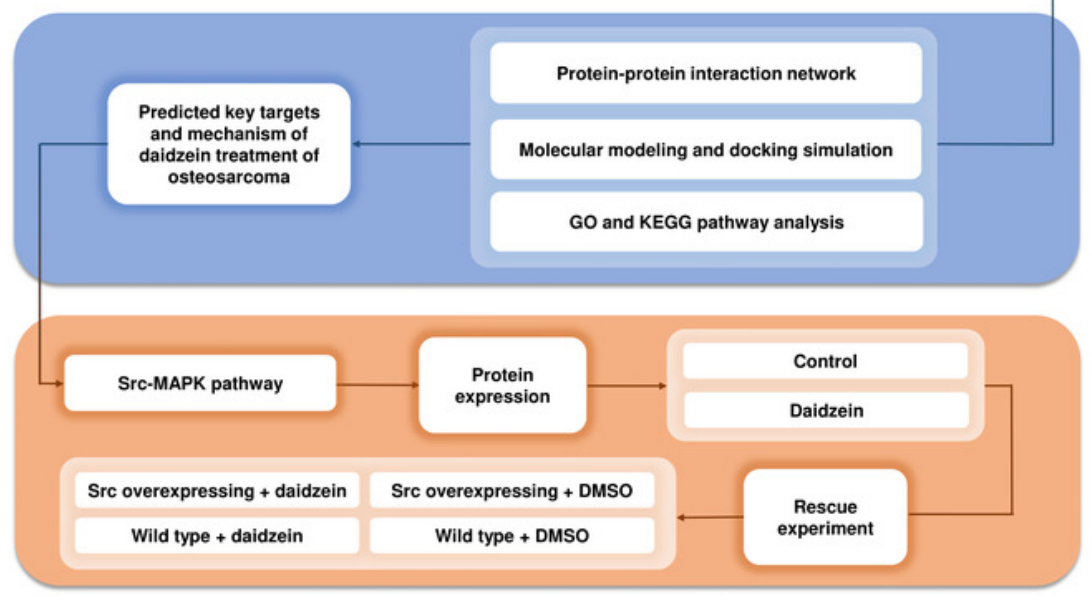




\section{Figure 2}

Daidzein inhibited cell proliferation and induced apoptosis in vitro.

(A) MTT analysis of 143B and U2OS cells treated with different concentrations of daidzein for 48 or $72 \mathrm{~h}$. MTT assay was performed in sextuplicate. Dose- and time-dependent inhibition of cell growth could be observed $\left(P<0.0001\right.$, ANOVA analysis ). (B) $I C_{50}$ values in $143 B$ and U2OS cells after 48 or $72 \mathrm{~h}$ of daidzein treatment. (C) MTT analysis of hFOB cells treated with different concentrations of daidzein for 48 or $72 \mathrm{~h}$. (D) $\mathrm{IC}_{50}$ values in non-tumor cells $\mathrm{hFOB}$ after 48 or $72 \mathrm{~h}$ of daidzein treatment. (E) Seven-day colony formation assay of 143B and U2OS cells treated with different concentrations of daidzein. (F) Colony counts in 143B and U2OS cells treated with different concentrations of daidzein from four independent experiments. (G) Hoechst 33342 staining assay of 143B and U2OS cells treated with different concentrations of daidzein. (Solid bars and errors represent the mean \pm SEM. Individual values were shown as dots of different shapes. Student's t-test, ${ }^{*} p<0.05,{ }^{* *} p<0.01,{ }^{* *} p$ $<0.001,{ }^{* * * *} \mathrm{p}<0.0001$ versus control group.) 
A

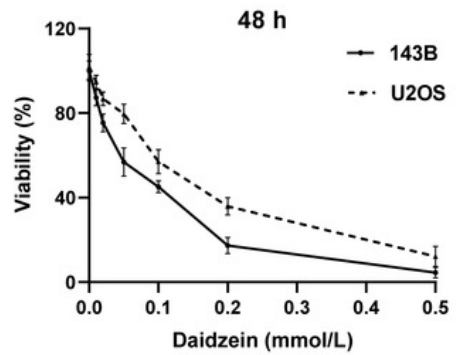

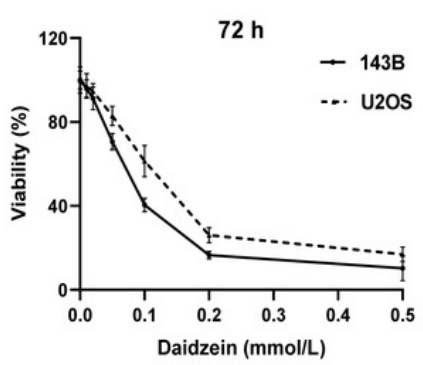

D
B

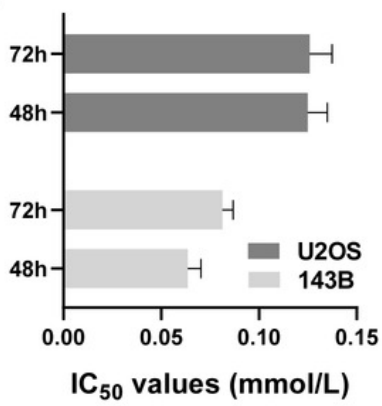

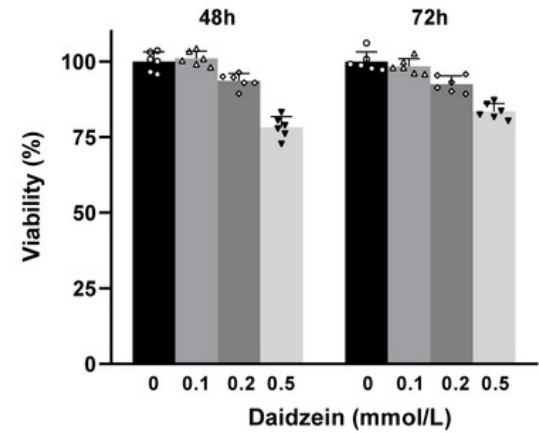

E
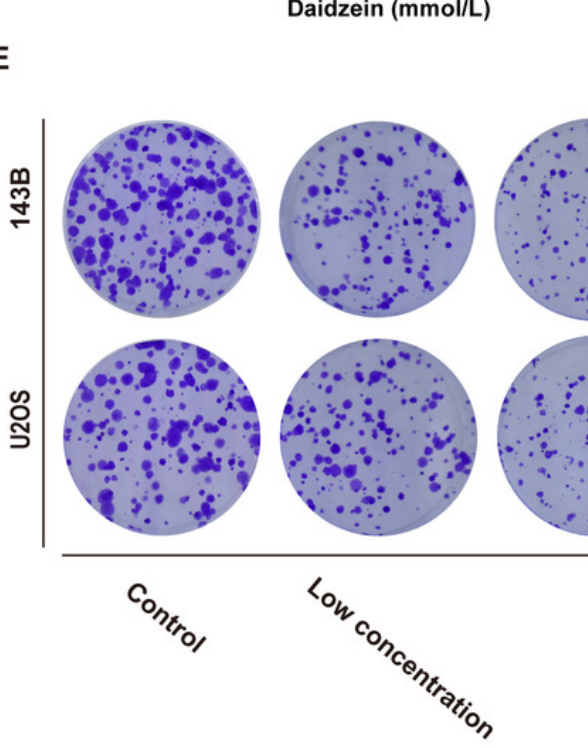

C

G

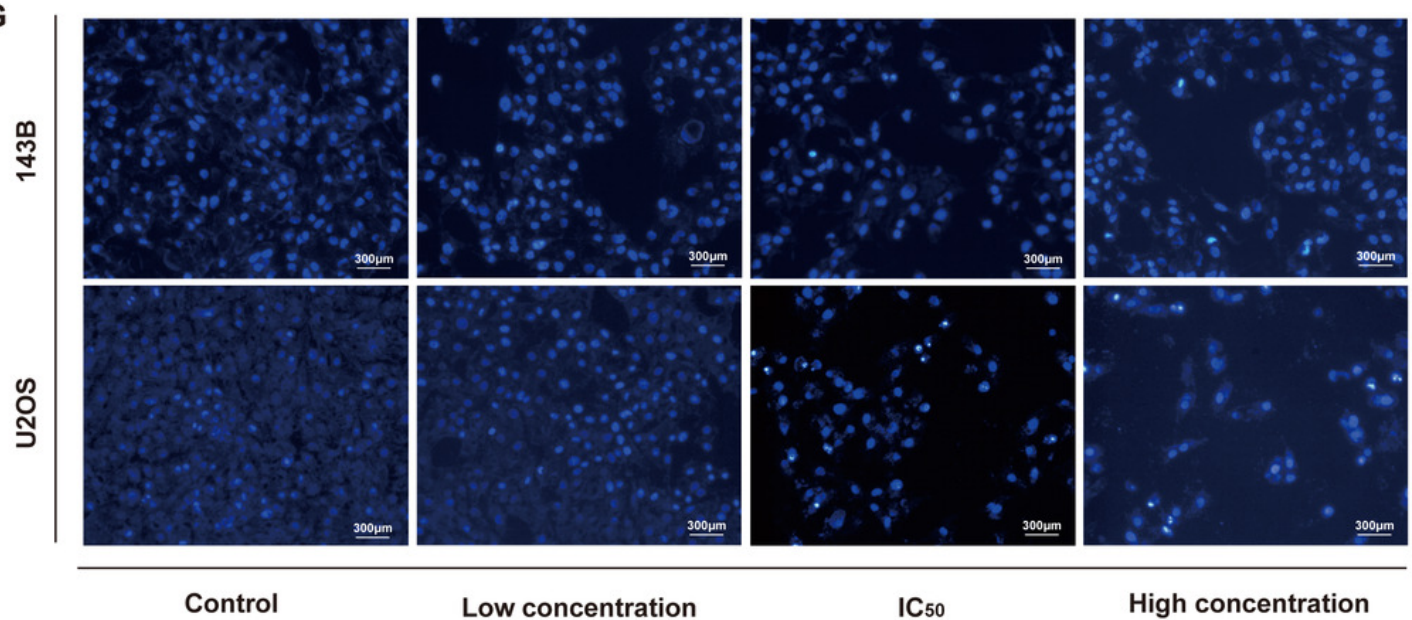

$\mathbf{F}$

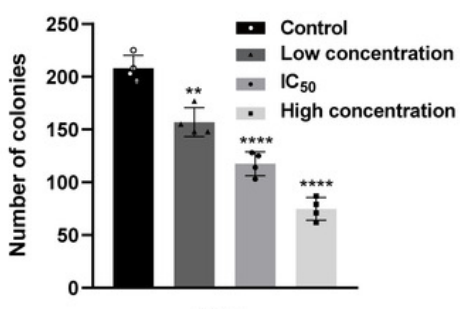

143B

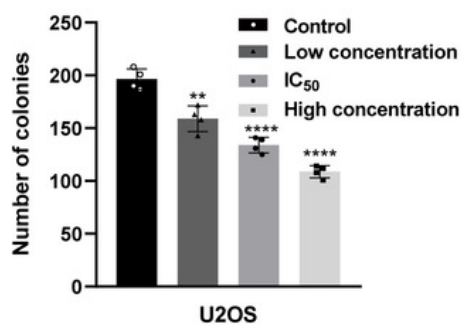

PeerJ reviewing PDF | (2021:03:59437:1:0:NEW 2 Jul 2021) 


\section{Figure 3}

Incubation of osteosarcoma cells with daidzein promoted apoptosis and cell cycle arrest when inhibited migration.

(A) 143B and U2OS cells treated with different concentrations of daidzein were stained with Annexin V-FITC/Pl, analyzed by flow cytometry. Quantitative analysis of apoptotic cells from three independent experiments. (B) Cell cycle detection of 143B and U2OS cells treated with different concentrations of daidzein, examined by flow cytometry. (C) Quantitative analysis of cycle distribution from three independent experiments. (D) Transwell assay of 143B and U2OS cells pretreated with different concentrations of daidzein. F) Invaded cell counts in 143B and U2OS cells pretreated with different concentrations of daidzein. (E) Scratch wound migration analysis of osteosarcoma cells treated with different concentrations of daidzein. (F) Wound closure rates in osteosarcoma cells treated with different concentrations of daidzein. (Solid bars and errors represent the mean \pm SEM, Individual values were shown as dots of different shapes, Student's t-test, ${ }^{*} p<0.05,{ }^{* *} p<0.01,{ }^{* * *} p<0.001,{ }^{* * * *} p<0.0001$ versus control group.) 
A

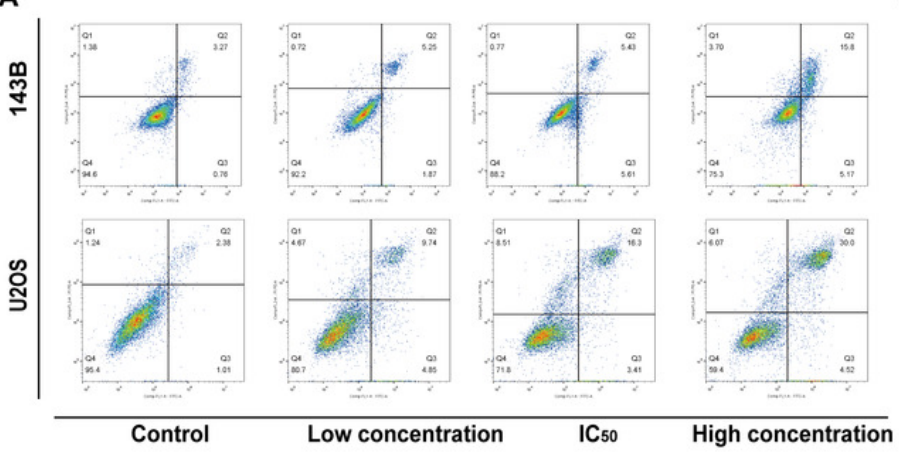

B

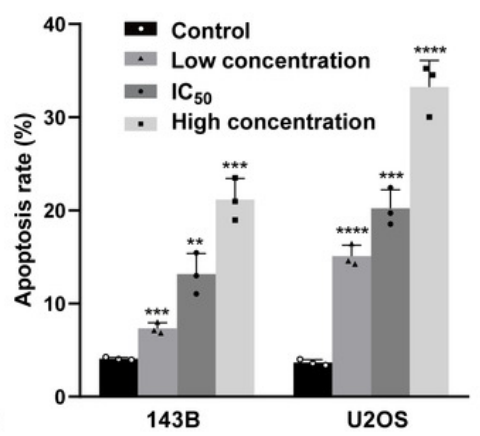

C

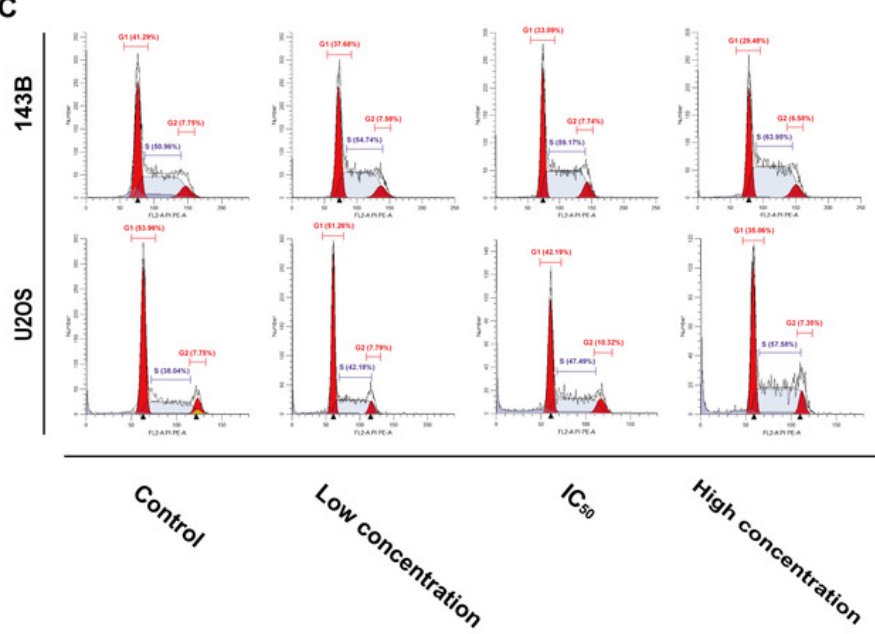

\section{D}

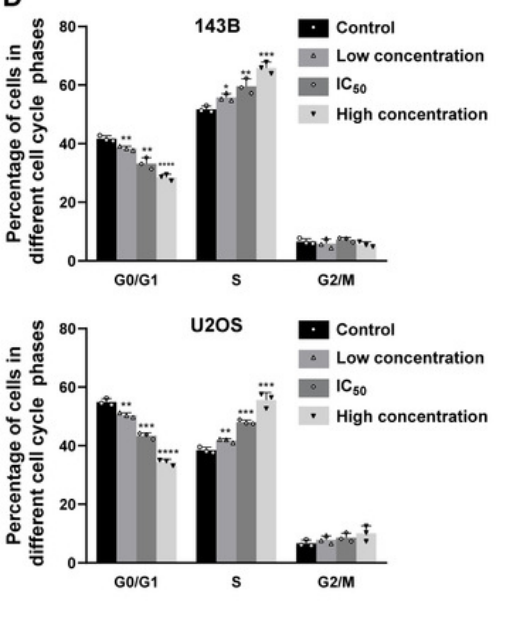

F
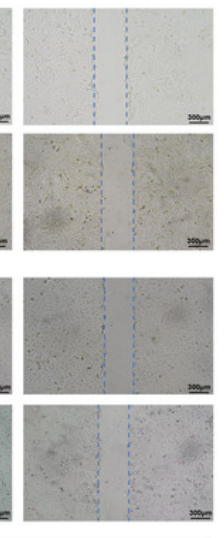

High concentration

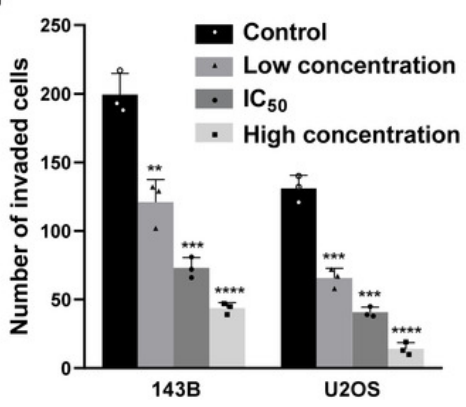

H

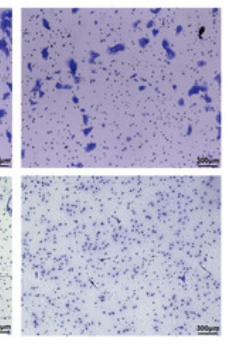

High concentration

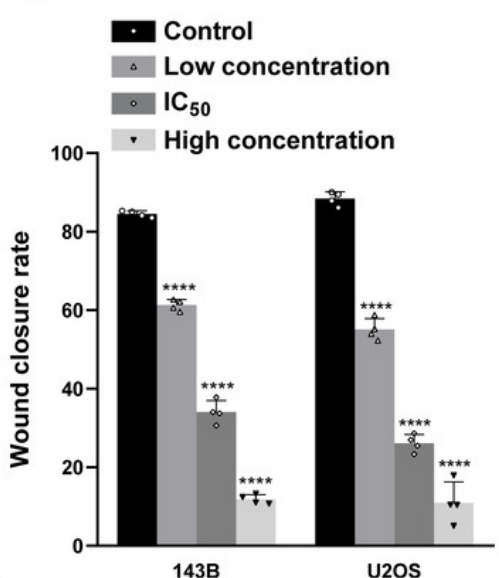

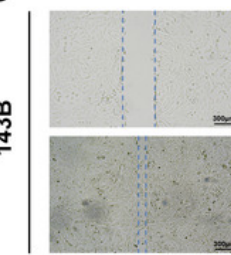

:

Control
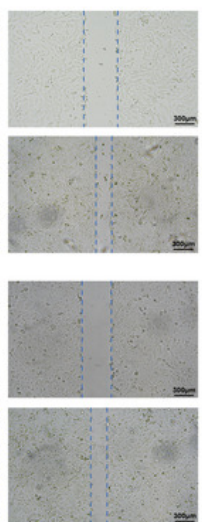

Low concentration
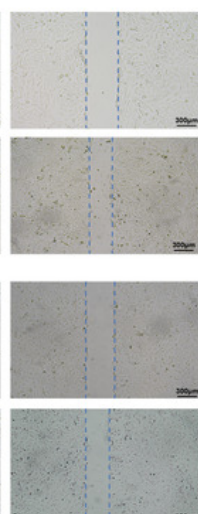

$\mathrm{IC}_{50}$ 


\section{Figure 4}

Daidzein exerts antitumor effects in vivo without obvious toxicity.

(A) Gross observation of osteosarcoma xenograft mice on the day the mice were sacrificed.

(B) Body weight during the daidzein injection. There was no significant difference between the two groups. (ANOVA analysis) (C) Gross observation of tumor tissue on the day the mice were sacrificed. (D) Tumor volume during the daidzein injection. Time-dependent inhibition of cell growth could be observed ( $P<0.0001$, ANOVA analysis) (E) H\&E staining of the tumor. The daidzein group showed obvious tumor necrosis. (F) Tumor weight on day 16. Solid bars and errors represent the mean \pm SEM, Student's t-test, ${ }^{* * *} p<0.0001$ versus control group. 
A

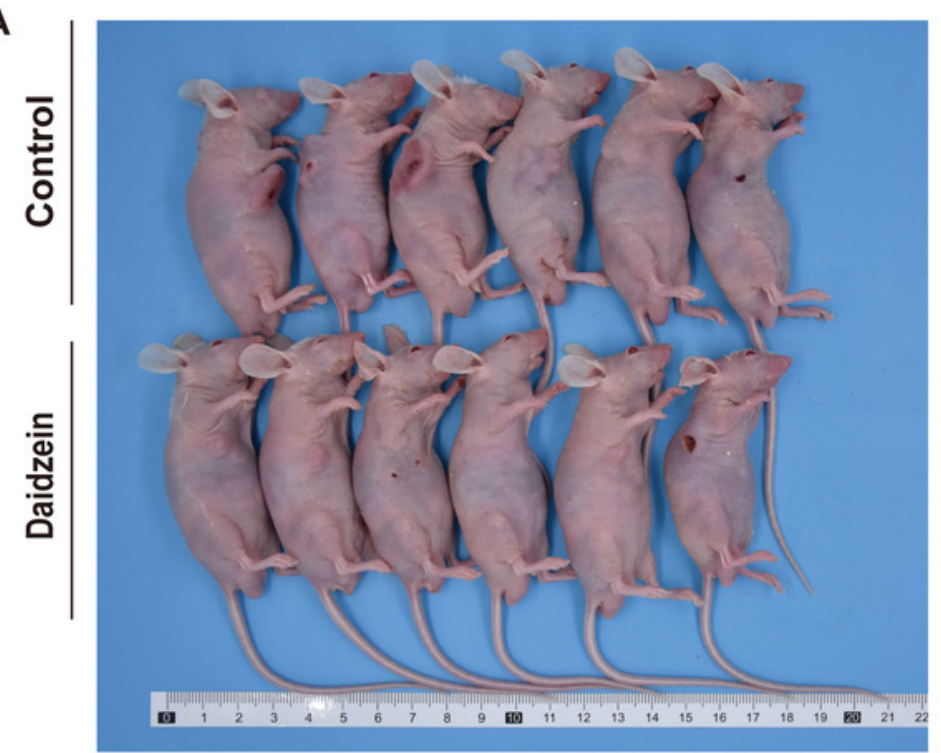

C

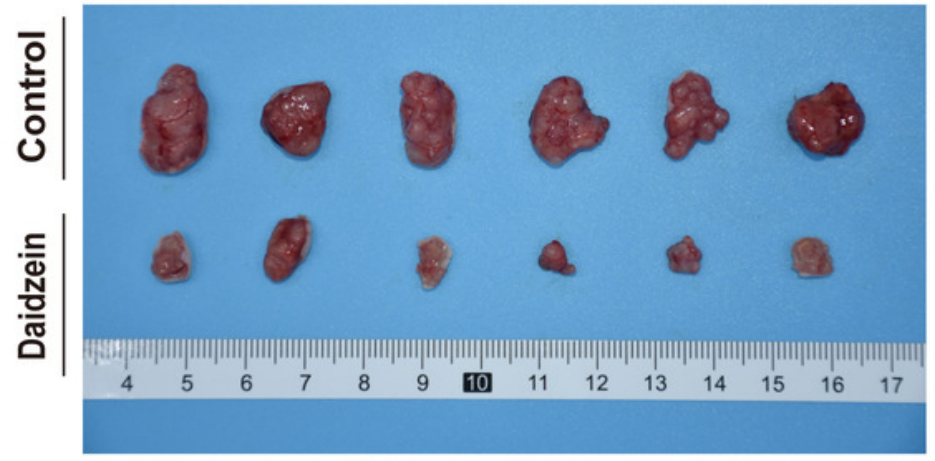

E

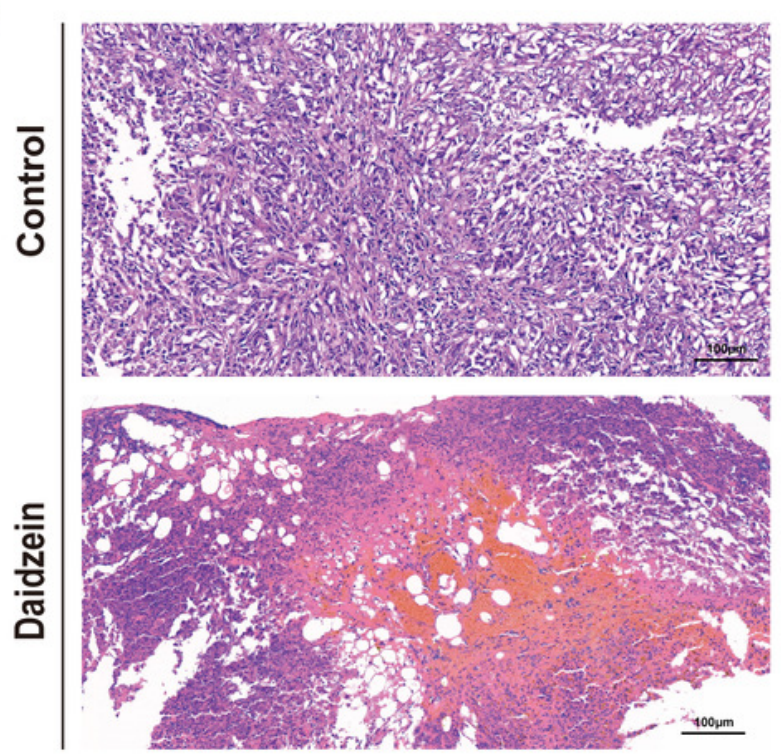

$\mathbf{F}$
B

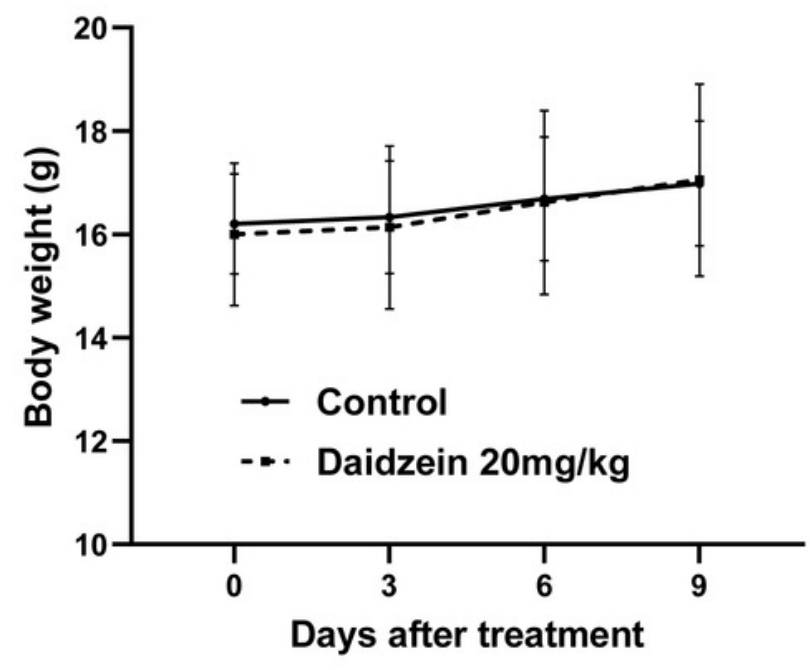

D

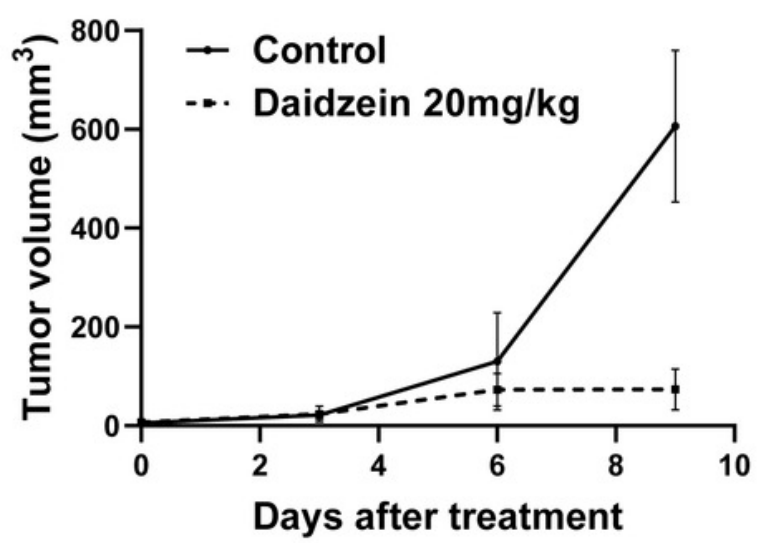

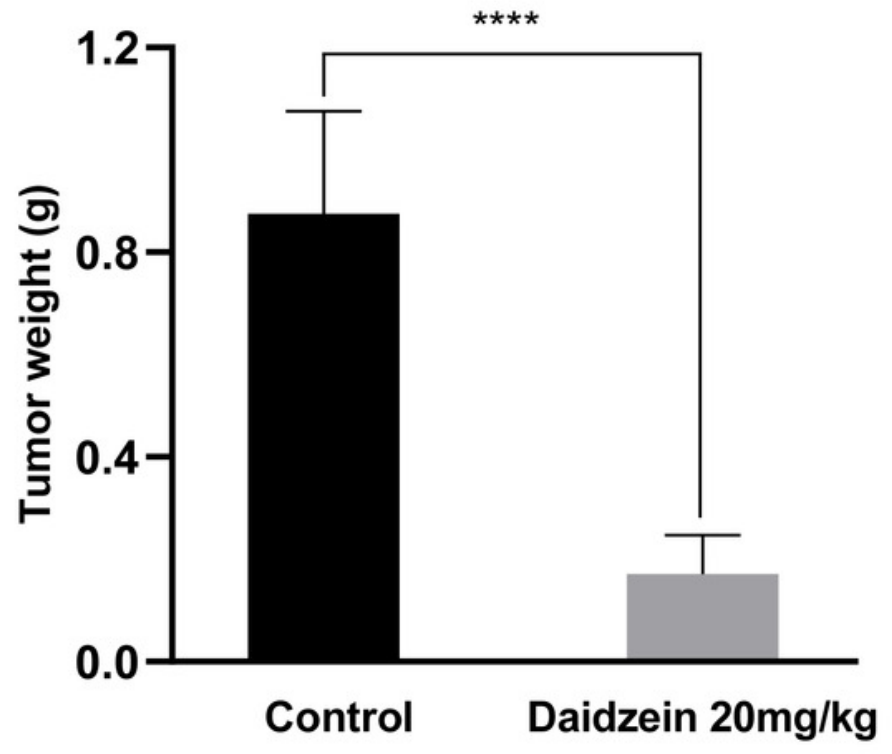




\section{Figure 5}

Src is the most likely target molecule for direct binding by daidzein.

(A) Network of daidzein-target genes. Purple diamonds represent daidzein; green circles represent target genes of daidzein. (B) Venn diagram revealing the common target genes of daidzein against osteosarcoma. (C) Protein-protein interaction (PPI) network. Nodes: common targets of daidzein and osteosarcoma; Edges: PPIs between targets of daidzein and their interaction partners. (D) The top 30 hub genes in the PPI network that interacted with the most numerous edges. (E) The Ball and Stick 3D structure of daidzein. The gray ball represents the carbon atom when the red ball represents the oxygen atom, and the small white ball represents the hydrogen atom. (F) Molecular docking simulation predicted the amino acid residues bound by daidzein. (G) Molecular docking simulation of daidzein to Src protein. Sticks represent the three-dimensional structure of daidzein; the surface represents the protein structure of Src protein. 
A

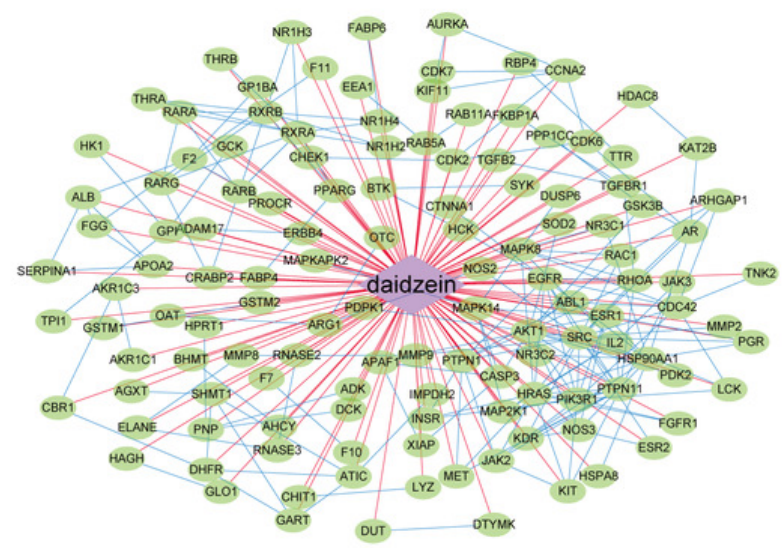

C

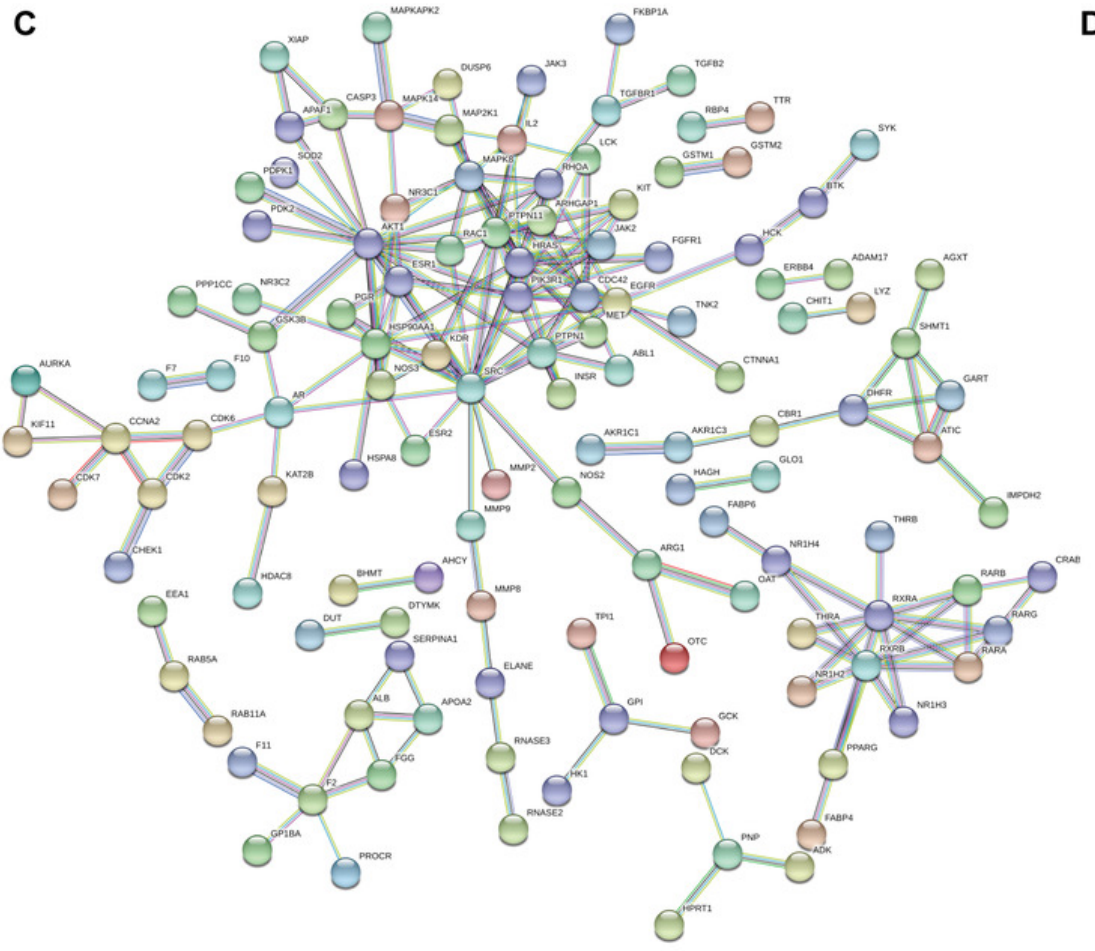

\section{B}

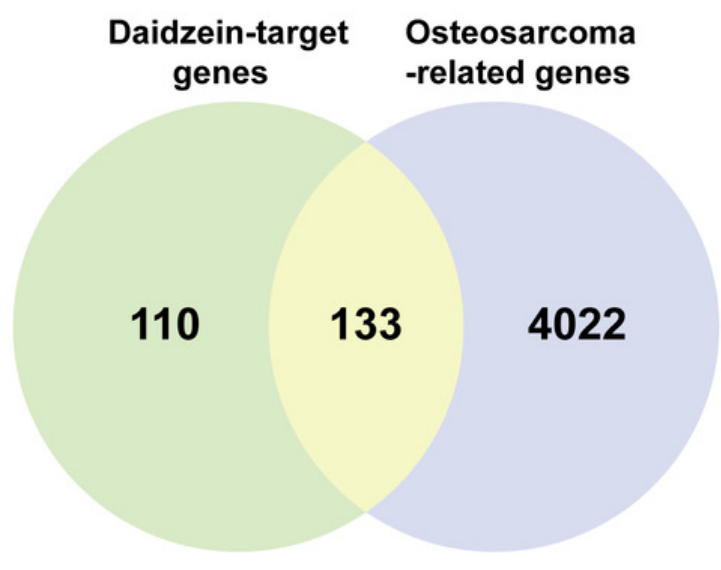

D

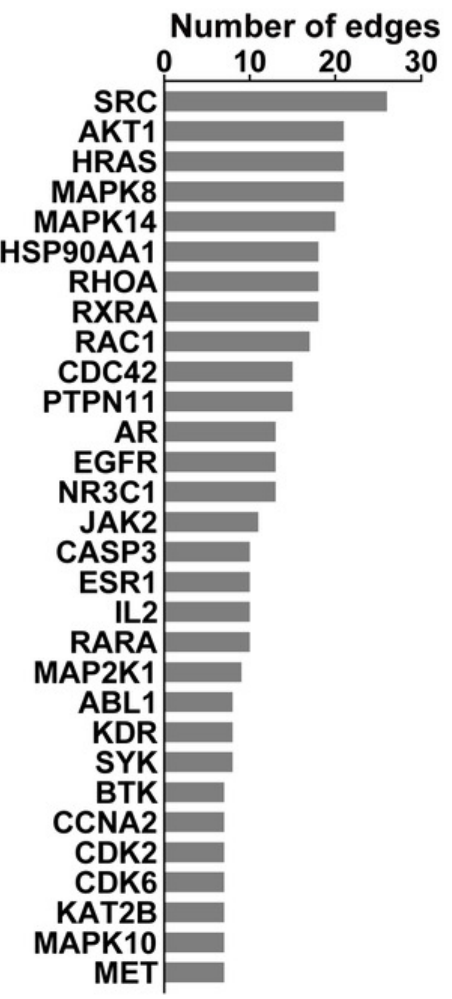

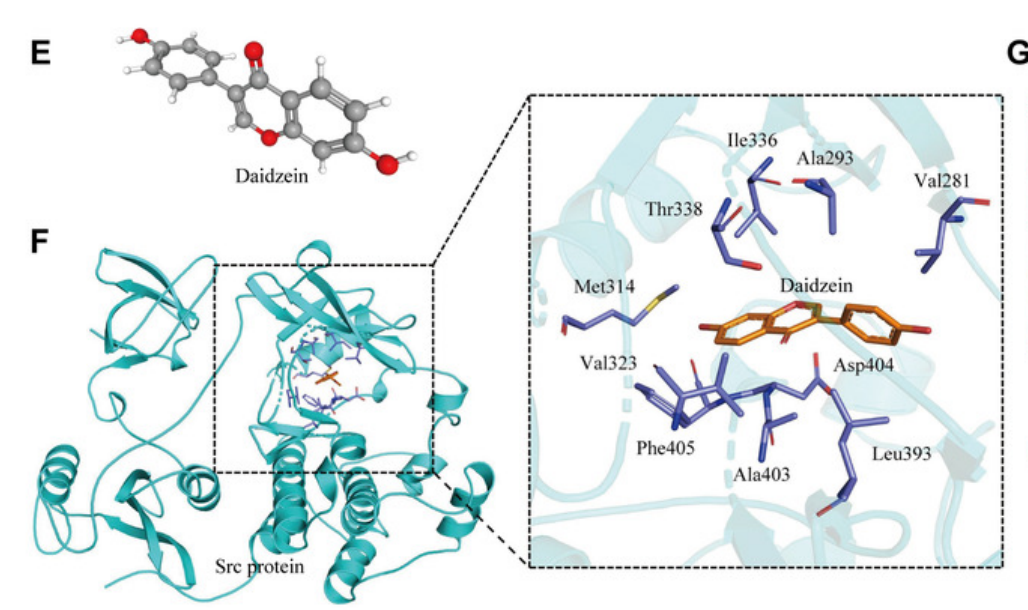

G

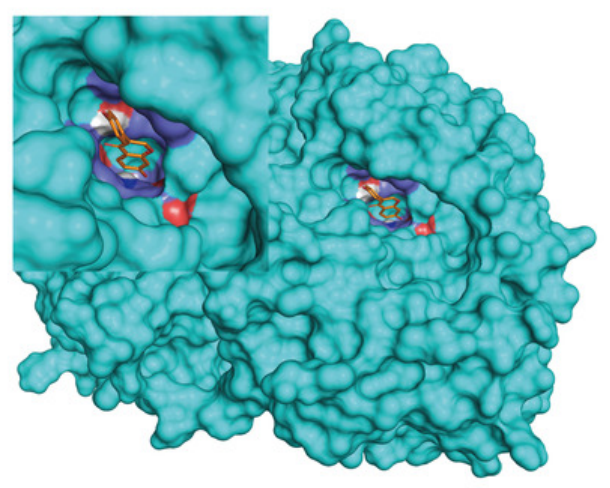




\section{Figure 6}

Daidzein inhibits phosphorylation of Src and ERK in osteosarcoma cells.

(A) The top 20 highly enriched functional terms analyzed by GO enrichment. (B) The top 20 highly enriched signaling pathways analyzed by KEGG enrichment. (C) Western blot results of the Src-MAPK pathway in 143B and U2OS cells treated with different concentrations of daidzein. (D) Quantitative analysis of western blot. Solid bars and errors represent the mean \pm SEM, Individual values were shown as dots of different shapes. Student's t-test, ${ }^{*} p<0.05$, ${ }^{* *} p<0.01$ versus control group. 
A

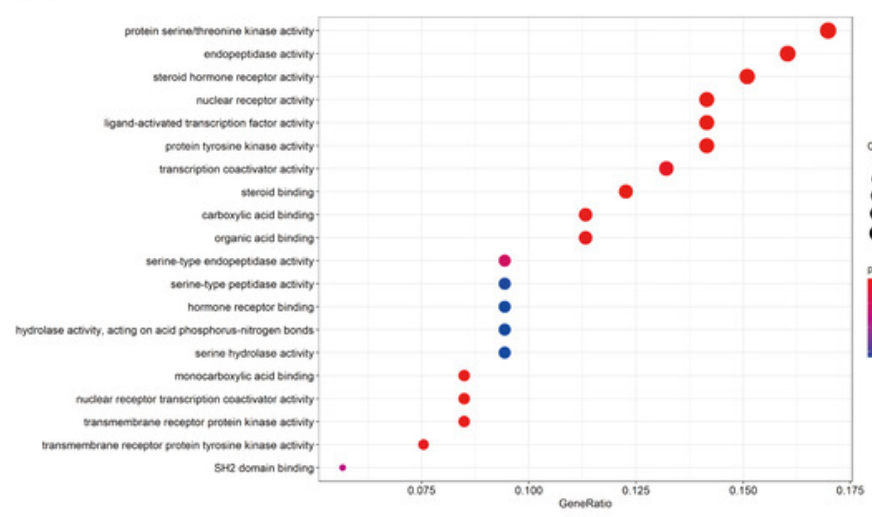

C

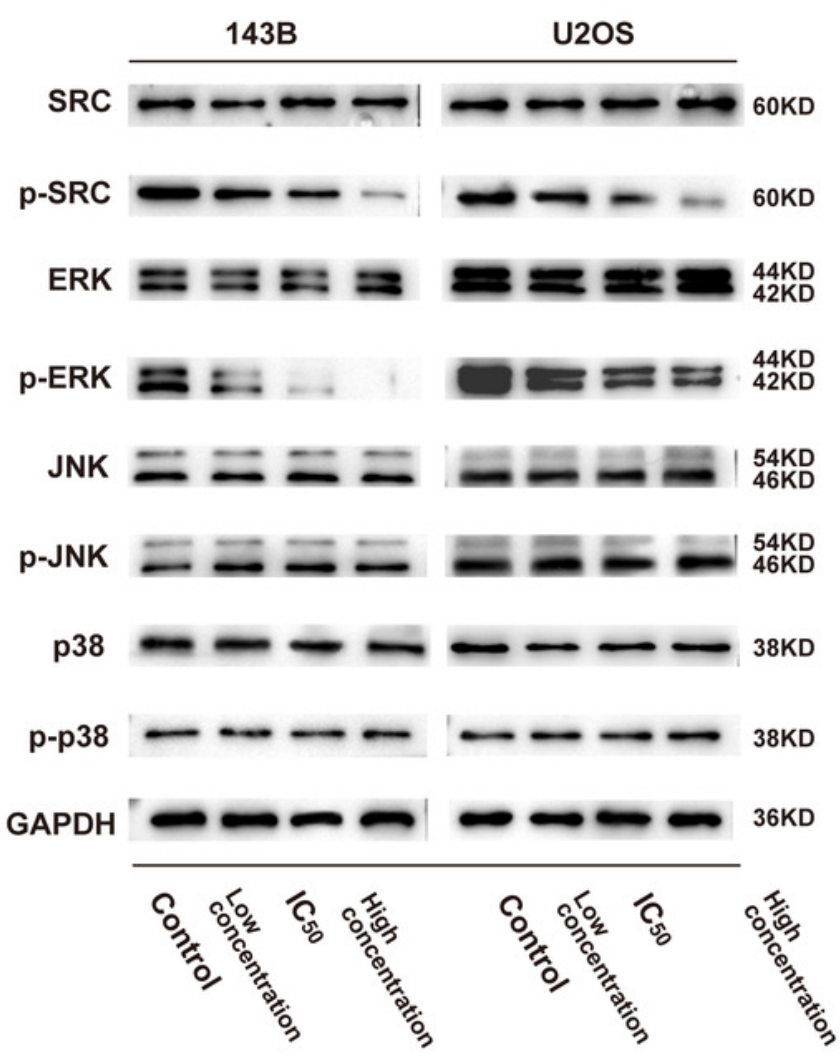

B

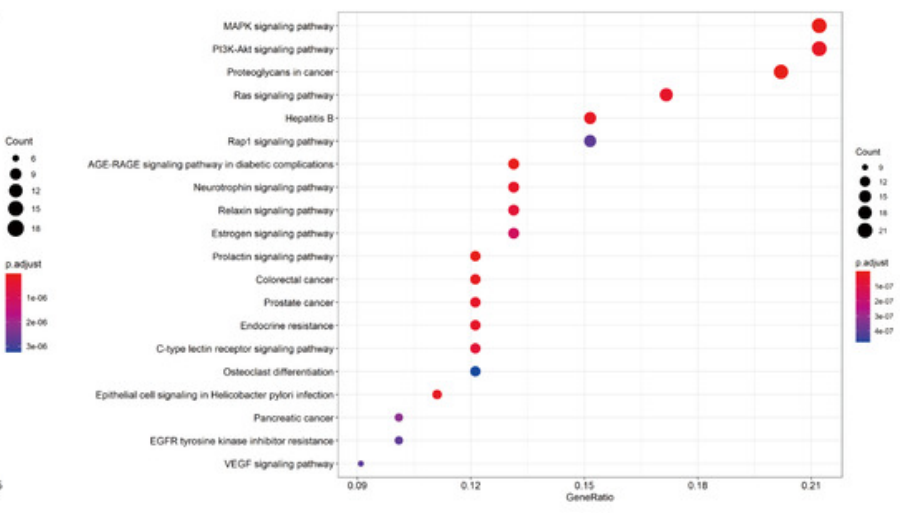

D
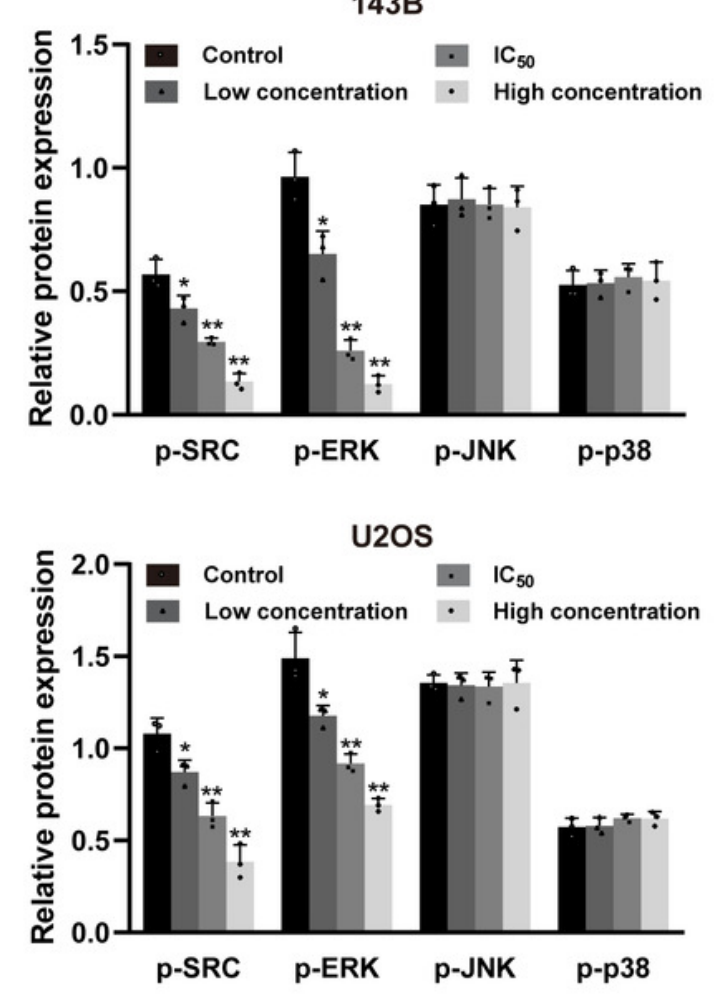


\section{Figure 7}

Daidzein exerts antitumor effects through the Src-ERK pathway in osteosarcoma confirmed by the Src overexpression rescue experiments.

(A) The expression of the green fluorescent protein EGFP in Src-overexpressing 143B and U2OS cells was observed under a fluorescence microscope. (B) Western blot results of the Src-ERK pathway in the Src overexpression rescue experiments. (C) Quantitative analysis of western blot of the Src overexpression rescue experiments. (D) MTT analysis of the SrC overexpression rescue experiments. (E) Apoptotic cells were detected by the V-FITC/PI double staining assay in the Src overexpression rescue experiments. (F) Quantitative analysis of apoptotic cells from three independent experiments. (Solid bars and errors represent the mean \pm SEM, Individual values were shown as dots of different shapes. Student's t-test, ${ }^{*} \mathrm{p}<$ $0.05,{ }^{* *} p<0.01,{ }^{* * *} p<0.001$ versus control group.) 
A

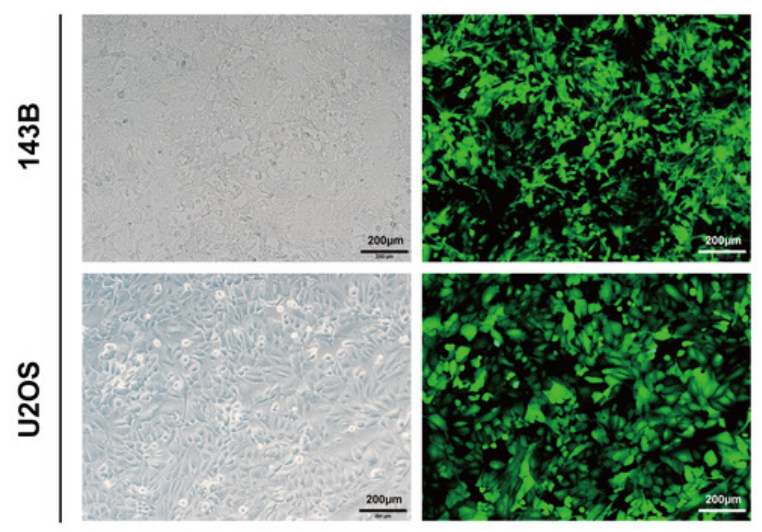

B

B $\frac{\text { U2OS }}{-\ldots-\infty-\infty{ }_{60 \mathrm{KD}}}$ p-SRC - - - - - - - $60 \mathrm{KD}$ $\mathrm{ERK}=z=z=2={ }_{42 \mathrm{KD}}^{44 \mathrm{KD}}$

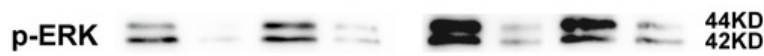
GAPDH $-\infty-\infty \infty \infty_{36 \mathrm{KD}}$

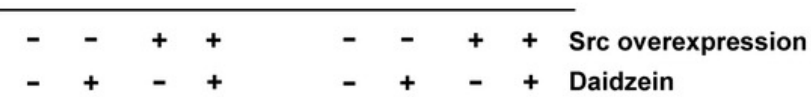

C 143B

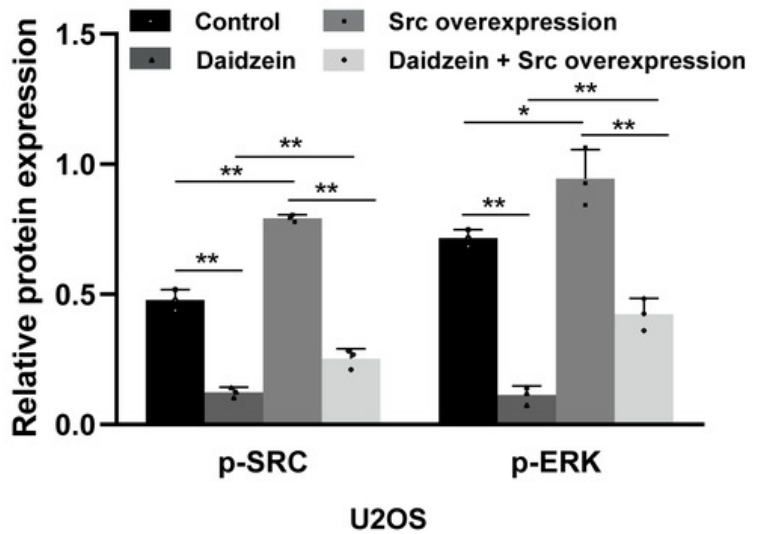

. Control 1. Daidzein .. Src overexpression

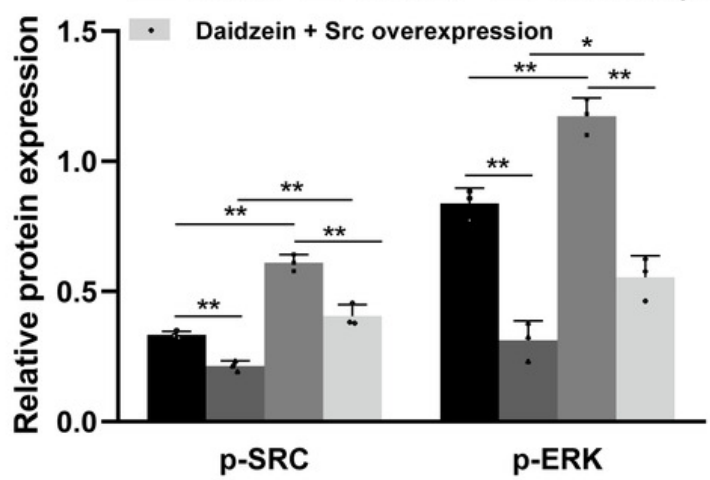

D

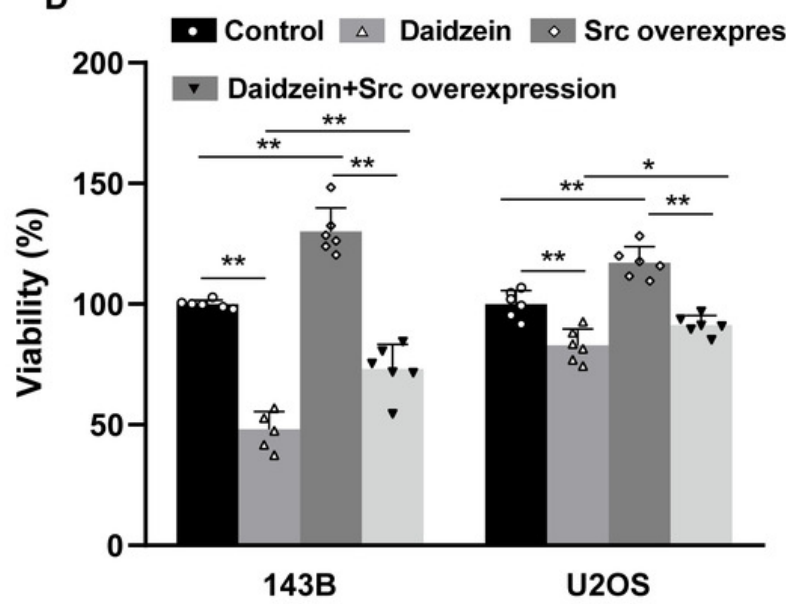

E

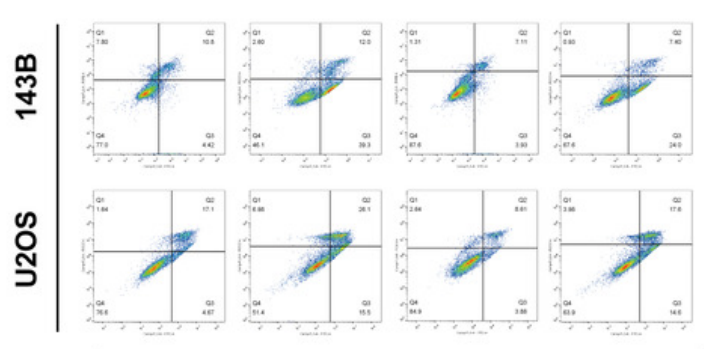

$\mathbf{F}$

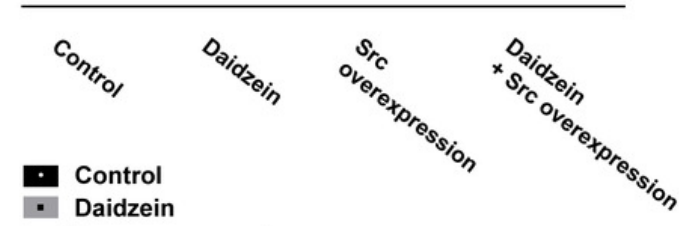

- Daidzein

- Src overexpression

- Daidzein + Src overexpression

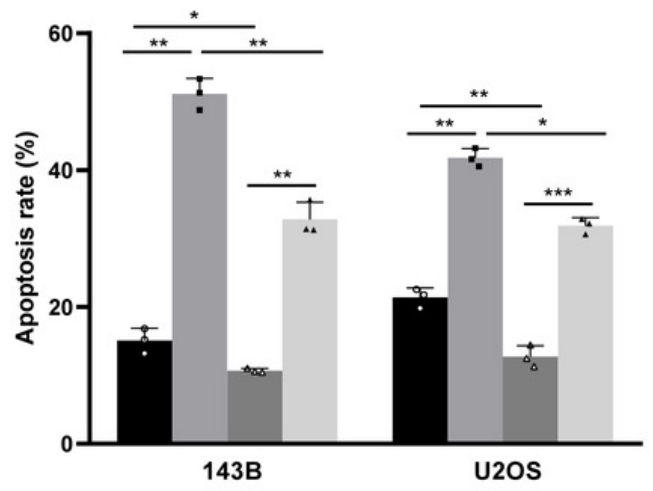

